Хромов А. П. Функционально-дифференциальный оператор с инволюцией // Докл. РАН. 2007. Т. 414, № 4. C. $1309-1312$.

4. Бурлуизкая М. Ш., Хромов А. П. Об одной теореме равносходимости на всем отрезке для функциональнодифференциальных операторов // Изв. Сарат. ун-та. Нов. сер. Сер. Математика. Механика. Информатика. 2009. Т. 9, вып. 4. С. 3-10.

\title{
Jordan-Dirichlet Theorem for Functional Differential Operator with Involution
}

\section{Sh. Burlutskaya}

Voronezh State University, Russia, 394006, Voronezh, Universitetskaya pl., 1, bmsh2001@mail.ru

In this paper the problem of decomposability of a function $f(x)$ into Fourier series with respect to the system of eigenfunctions of a functional-differential operator with involution $L y=y^{\prime}(1-x)+\alpha y^{\prime}(x)+p_{1}(x) y(x)+p_{2}(x) y(1-x), y(0)=\gamma y(1)$ is investigated. Based on the study of the resolvent of the operator easier and using the method of contour integration of the resolvent, we obtain the sufficient conditions for the convergence of the Fourier series for a function $f(x)$ (analogue of the Jordan-Dirichlet's theorem).

Key words: functional-differential operator, involution, equiconvergence, Fourier series.

\section{References}

1. Khromov A. P. Equiconvergence theorems for integrodifferential and integral operators. Mathematics of the USSR-Sbornik, 1982, vol. 42, no. 3, pp. 331-355.

2. Khromov A. P. Inversion of integral operators with kernels discontinuous on the diagonal. Math. Notes, 1998, vol. 64 , no. 5-6, pp. 804-813. DOI: $10.4213 / \mathrm{mzm} 1472$.

3. Burlutskaya M. Sh., Kurdyumov V. P., Lukonina A. S., Khromov A. P. A functional-differential operator with involution. Doklady Math., 2007, vol. 75, no 3, pp. 399402.

4. Burlutskaya M. Sh., Khromov A. P. On the same theorem on a equiconvergence at the whole segment for the functional-differential operators. Izv. Sarat. Univ. N.S. Ser. Math. Mech. Inform., 2009, vol. 9, iss. 4, pt. 1, pp. 3-10 (in Russian).

УДК 501.1

\section{КОГОМОЛОГИИ АЛГЕБРЫ ЛИ ВЕКТОРНЫХ ПОЛЕЙ НЕКОТОРОГО ОДНОМЕРНОГО ОРБИФОЛДА}

\section{Е. Ю. Волокитина}

Ассистент касредры геометрии, Саратовский государственный университет им. Н. Г. Чернышевского, evgenia.yu@gmail.com

И. М. Гельсранд и Д. Б. Фукс доказали, что когомологии алгебры Ли векторных полей на окружности изоморфрны тензорному произведению кольца полиномов с одной образуюшей степени 2 и внешней алгебры с одной образующей степени 3. В настоящей статье изучаются когомологии алгебры Ли векторных полей одномерного орбифолда $S^{1} / \mathbb{Z}_{2}$, который представляет собой пространство орбит при действии группы $\mathbb{Z}_{2}$ на окружности отражением относительно оси $O x$. Доказано, что рассматриваемые когомологии изоморсрны тензорному произведению внешней алгебры с двумя образующими степени 1 и кольца полиномов с одной образующей степени 2. В доказательстве используется метод Гельсранда-Фукса с модисрикациями для данного случая.

Ключевые слова: орбифролд, алгебра Ли, когомологии.

\section{ВВЕДЕНИЕ}

Пусть $S^{1}$ - единичная окружность в плоскости комплексного переменного $z, t-$ угловой параметр на окружности. Обозначим через $X=S^{1} / \mathbb{Z}_{2}$ орбифолд, получающийся из окружности, действием группы $\mathbb{Z}_{2}$, порожденной отражением относительно оси $O x . S^{1} / \mathbb{Z}_{2}-$ один из естественных одномерных орбифолодов. У данного орбифолда существует две особые точки, соответствующие значениям углового параметра $t=0$ и $t=\pi$. Обозначим через $\mathscr{U}\left(S^{1}\right)$ и $\mathscr{U}(X)$ алгебры Ли гладких векторных полей на окружности и орбифолде $X$ соответственно. Под гладкостью здесь и далее будем понимать гладкость класса $C^{\infty}$. Алгебра Ли $\mathscr{U}\left(S^{1}\right)$ - топологическая алгебра Ли с $C^{\infty}$-топологией, $\mathscr{U}(X)-$ ее замкнутая подалгебра. 
Каждая гладкая функция на орбифолде $X$ определяется четной периодической гладкой функцией на $\mathbb{R}$. Векторными полями на $X$ являются дифференцирования алгебры гладких функций на $X$.

Любое векторное поле на окружности представляется в виде $\xi(t) \frac{d}{d t}$, где $\xi(t)-$ гладкая периодическая функция. Будем называть векторные поля, для которых функция $\xi(t)$ нечетная(четная), нечетными(четными) векторными полями. Аналогичным образом векторное поле на орбифолде $X$ можно представить в виде $\xi(t) \frac{d}{d t}$, где $\xi(t)-$ нечетная периодическая гладкая функция. Поэтому под алгеброй Ли $\mathscr{U}(X)$ можно понимать алгебру Ли нечетных векторных полей на окружности. В дальнейшем будем вместо $\xi(t) \frac{d}{d t}$ писать просто $\xi(t)$.

Определение 1. q-мерной коцепью топологической алгебры Ли $\mathfrak{g}$ с коэффициентами в тривиальном $\mathfrak{g}$-модуле $\mathbb{R}$ называется непрерывный кососимметрический $q$-линейный функционал на $\mathfrak{g}$ со значениями в $\mathbb{R}$.

Дифференциал задается формулой: если $L \in C^{q}(\mathfrak{g})$, то

$$
d L\left(\xi_{1}, \ldots, \xi_{q+1}\right)=\sum_{1 \leq i<j \leq q+1}(-1)^{i+j-1} L\left(\left[\xi_{i}, \xi_{j}\right], \xi_{1}, \ldots, \widehat{\xi}_{i}, \ldots, \widehat{\xi}_{j}, \ldots, \xi_{q+1}\right) .
$$

Произведение коцепей определяется следующим образом: если $L_{1} \in C^{q}(\mathfrak{g}), L_{2} \in C^{r}(\mathfrak{g})$, то $L_{1} \wedge L_{2} \in C^{q+r}(\mathfrak{g})$ и

$$
\begin{aligned}
L_{1} \wedge L_{2}\left(\xi_{1}, \ldots, \xi_{q+r}\right) & =\sum_{1 \leq i_{1}<\ldots<i_{r} \leq q+r}(-1)^{i_{1}+\ldots+i_{q}-\frac{q(q-1)}{2}} \cdot L_{1}\left(\xi_{i_{1}}, \ldots, \xi_{i_{q}}\right) \times \\
& \times L_{2}\left(\xi_{1}, \ldots, \widehat{\xi_{i_{1}}}, \ldots, \widehat{\xi_{i_{q}}}, \ldots, \xi_{q+r}\right) .
\end{aligned}
$$

Обозначим через $C^{*}\left(\mathscr{U}\left(S^{1}\right)\right)$ и $C^{*}(\mathscr{U}(X))$ комплексы непрерывных коцепей с коэффициентами в тривиальном представлении $\mathbb{R}$ для алгебр Ли $\mathscr{U}\left(S^{1}\right)$ и $\left.\mathscr{U}(X)\right)$ соответственно. В статье [1] были вычислены одномерные и двумерные когомологии для алгебры Ли $\mathscr{U}(X)$. Сформулируем этот результат.

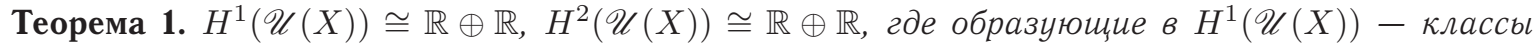
коциклов

$$
\alpha_{1}(\xi(t))=\frac{d \xi}{d t}(0), \quad \alpha_{2}(\xi(t))=\frac{d \xi}{d t}(\pi),
$$

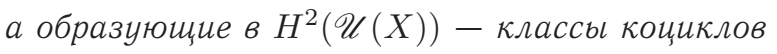

$$
\beta(\xi(t), \eta(t))=\int_{0}^{\pi}\left(\frac{d^{2} \xi}{(d t)^{2}} \frac{d \eta}{d t}-\frac{d \xi}{d t} \frac{d^{2} \eta}{(d t)^{2}}\right) d t
$$

$u \alpha_{1} \wedge \alpha_{2}$.

Основным результатом настоящей статьи является следующая теорема.

Теорема 2. Алгебра когомологий $H^{*}(\mathscr{U}(X)) \cong S(\widetilde{\beta}) \otimes \bigwedge\left(\widetilde{\alpha}_{1}, \widetilde{\alpha}_{2}\right)$, где $\widetilde{\beta}, \widetilde{\alpha}_{1}, \widetilde{\alpha}_{2}-$ классы коциклов $\beta, \alpha_{1}, \alpha_{2}$, определенных формулами (2) и (3). (\( $\left(\widetilde{\alpha}_{1}, \widetilde{\alpha}_{2}\right)$ обозначает внешнюю алгебру с двумя образующими, а $S(\widetilde{\beta})-$ кольцо многочленов с одной образующей.)

Сформулируем для сравнения аналогичный результат для окружности, доказанный в [2].

Теорема 3. Алгебра когомологий $H^{*}\left(\mathscr{U}\left(S^{1}\right)\right) \cong S\left(\widetilde{\beta}_{1}\right) \otimes \bigwedge\left(\widetilde{\alpha_{3}}\right)$, где $\widetilde{\beta}_{1}, \widetilde{\alpha}_{3}-$ классы коциклов $\beta_{1}$ и $\alpha_{3}$, которые определяются формулами

$$
\beta_{1}(\xi, \eta)=\int_{0}^{2 \pi}\left(\xi^{\prime \prime} \eta^{\prime}-\xi^{\prime} \eta^{\prime \prime}\right) d t, \quad \alpha_{3}(\xi, \eta, \chi)=\int_{0}^{2 \pi}\left|\begin{array}{ccc}
\xi & \eta & \chi \\
\xi^{\prime} & \eta^{\prime} & \chi^{\prime} \\
\xi^{\prime \prime} & \eta^{\prime \prime} & \chi^{\prime \prime}
\end{array}\right| d t .
$$

Заметим, что коцикл $\beta_{1}$ при ограничении на подалгебру $\mathscr{U}(X)$ равен нулю, а коцикл $\alpha_{3}$ при ограничении на подалгебру когомологичен нулю и, следовательно, гомоморфизм ограничения $H^{*}\left(\mathscr{U}\left(S^{1}\right)\right) \rightarrow H^{*}(\mathscr{U}(X))$ тривиален.

Для вычисления когомологий $H^{*}(\mathscr{U}(X))$ воспользуемся методом Гельфанда-Фукса вычисления когомологий алгебры Ли гладких векторных полей на замкнутом гладком многообразии, описанным в [2], с некоторыми модификациями для нашего случая. 


\section{2. ФИЛЬТРАЦИЯ $C_{k}^{q}(X)$.}

Пусть р: $C^{*}\left(\mathscr{U}\left(S^{1}\right)\right) \rightarrow C^{*}(\mathscr{U}(X))$ - отображение ограничения коцепей на подалгебру. Оно является гомоморфизмом комплексов. Тогда

$$
C^{*}(\mathscr{U}(X)) \cong C^{*}\left(\mathscr{U}\left(S^{1}\right)\right) / \operatorname{ker} \mathrm{p},
$$

где ker р состоит из таких коцепей из $C^{*}\left(\mathscr{U}\left(S^{1}\right)\right.$, которые равны нулю, если все аргументы - нечетные векторные поля.

Рассмотрим также отображение і: $C^{*}(\mathscr{U}(X)) \rightarrow C^{*}\left(\mathscr{U}\left(S^{1}\right)\right)$, которое определяется следующим образом:

$$
\text { для } L \in C^{q}(\mathscr{U}(X)) \mathrm{i}(L)\left(\xi_{1}, \ldots, \xi_{q}\right)=L\left(\xi_{1}, \ldots, \xi_{q}\right),
$$

если все $\xi_{1}, \ldots, \xi_{q}$ - нечетные и $\mathrm{i}(L)\left(\xi_{1}, \ldots, \xi_{q}\right)=0$, если среди $\xi_{1}, \ldots, \xi_{q}$ есть хотя бы одно четное векторное поле.

Так как $d(\mathrm{i}(L)) \neq \mathrm{i}(d L)$, отображение і не будет гомоморфизмом комплексов, а только линейным непрерывным отображением векторных пространств. Таким образом $C^{*}(\mathscr{U}(X))$ можно вложить в $C^{*}\left(\mathscr{U}\left(S^{1}\right)\right.$ как векторное подпространство, но не как подкомплекс.

Обозначим через $\widetilde{d}$ следующий дифференциал комплекса $\mathrm{i}\left(C^{*}(\mathscr{U}(X))\right.$. Пусть $L \in \mathrm{i}\left(C^{q}(\mathscr{U}(X))\right)$, тогда $\widetilde{d} L=\mathrm{i}(\mathrm{p}(d L))$. Заметим также, что $\mathrm{p} \circ \mathrm{i}=\mathrm{id}_{C^{*}(\mathscr{U}(X))}$. Получаем следующий изоморфизм комплексов:

$$
\left(C^{*}(\mathscr{U}(X)), d\right) \cong\left(\mathrm{i}\left(C^{*}(\mathscr{U}(X))\right), \widetilde{d}\right),
$$

где і $\left(C^{*}(\mathscr{U}(X))\right)$ - подпространство в $C^{*}\left(\mathscr{U}\left(S^{1}\right)\right)$.

Пусть $M$ - замкнутое гладкое многообразие. Напомним, как в [2] строится фильтрация для $C^{q}(\mathscr{U}(M))$. Будем говорить, что коцепь $L \in C^{q}(\mathscr{U}(M))$ имеет фильтрацию $\leq k$, если из $L\left(\xi_{1}, \ldots, \xi_{q}\right) \neq 0$ следует, что существуют $k$ точек $x_{1}, \ldots, x_{k} \in M$ таких, что каждое из векторных полей $\xi_{1}, \ldots, \xi_{q}$ не обращается в нуль в окрестности хотя бы одной из точек $x_{1}, \ldots, x_{k}$. Обозначим через $C_{k}^{q}(M)$ подпространство пространства $C^{q}(\mathscr{U}(M))$, составленное из элементов фильтрации $\leq k$. Тогда

$$
0=C_{0}^{q}(M) \subset C_{1}^{q}(M) \subset \ldots \subset C_{q}^{q}(M)=C^{q}(\mathscr{U}(M)) .
$$

Аналогичным образом можно определить фильтрацию пространства $C^{q}(\mathscr{U}(X))$ подпространствами $C_{k}^{q}(X)$, просто заменив в вышестоящем определении фильтрации $M$ на $X$.

Воспользовавшись изоморфизмом (4), определим фильтрацию пространств $\mathrm{i}\left(C^{q}(\mathscr{U}((X)))\right.$ подпространствами $\widetilde{C}_{k}^{q}=\mathrm{i}\left(C_{k}^{q}(X)\right)$. Тогда $\widetilde{C}_{k}^{q} \cong C_{k}^{q}(X)$.

Рассмотрим также фильтрацию пространств $C^{q}\left(\mathscr{U}\left(S^{1}\right)\right)$ подпространствами $C_{k}^{q}\left(S^{1}\right)$, которые определены ранее, если в качестве $M$ рассмотреть $S^{1}$. Тогда $\widetilde{C}_{k}^{q} \cong C_{k}^{q}\left(S^{1}\right) \cap \mathrm{i}\left(C^{q}(\mathscr{U}((X)))\right.$, то есть $\widetilde{C}_{k}^{q}-$ подпространство в пространстве $C_{k}^{q}\left(S^{1}\right)$.

Справедлива следующая лемма.

Лемма 1. Образ пространства $C_{k}^{q}(X)$ при гомоморфизме d содержится в $C_{k}^{q+1}(X)$.

Доказательство. Пусть $L \in C_{k}^{q}(X)$ и $d L\left(\xi_{1}, \ldots, \xi_{q+1}\right) \neq 0$. Тогда из определения гомоморфизма $d$ (1) следует, что $L\left(\left[\xi_{i}, \xi_{j}\right], \xi_{1}, \ldots, \widehat{\xi}_{i}, \ldots, \widehat{\xi}_{j}, \ldots, \xi_{q+1}\right) \neq 0$ для некоторых $1 \leq i<j \leq q+1$. Это означает, что существуют такие точки $t_{1}, \ldots, t_{k} \in X$, что каждое из векторных полей $\left[\xi_{i}, \xi_{j}\right], \xi_{1}, \ldots, \widehat{\xi}_{i}, \ldots, \widehat{\xi}_{j}, \ldots, \xi_{q+1}$ не равно нулю в окрестности хотя бы одной из этих точек. Каждое из векторных полей $\xi_{i}, \xi_{j}$ отличается от нулевого в любой окрестности, в которой отличается от нулевого векторное поле $\left[\xi_{i}, \xi_{j}\right]$. Поэтому векторные поля $\xi_{1}, \ldots, \xi_{q+1}$ не равны нулю в окрестности хотя бы одной из точек $t_{1}, \ldots, t_{k}$. Следовательно, $d L \in C_{k}^{q+1}$.

Из леммы 1 следует, что подпространсва $C_{k}^{q}(X)$ образуют подкомплекс комплекса $\left.\left(C^{q} \mathscr{U}(X)\right), d\right)$. В силу изоморфизма (4) подпространства $\widetilde{C}_{k}^{q}$ тоже образуют подкомплекс комплекса $\left.\left(\mathrm{i}\left(C^{q} \mathscr{U}(X)\right)\right), \widetilde{d}\right)$ и подкомплексы $\left(C_{k}^{q}(X), d\right)$ и $\left(\widetilde{C}_{k}^{q}, \widetilde{d}\right)$ изоморфны.

Для комплекса $\left(C^{*}\left(\mathscr{U}\left(S^{1}\right)\right), d\right)$ подпространство $C_{k}^{*}\left(S^{1}\right)$ с дифференциалом $d$ также является подкомплексом [2]. Подкомплексы $\widetilde{C}_{1}^{*}, C_{1}^{*}(X)$ и $C_{1}^{*}\left(S^{1}\right)$ называются диагональными. Обозначим их через $\widetilde{C}_{\triangle}^{*}, C_{\triangle}^{*}(X)$ и $C_{\triangle}^{*}\left(S^{1}\right)$. Пространство коцепей $\widetilde{C}_{\triangle}^{*}-$ подпространство в пространстве $C_{\triangle}^{*}\left(S^{1}\right)$.

В дальнейшем нам понадобится следующая лемма.

Лемма 2. При $q \geq 3$, если $\widetilde{d} L \in \widetilde{C}_{q-1}^{q+1}$, то $L \in \widetilde{C}_{q-1}^{q}$. В частности, всякий $q$-мерный коциикл при $q \geq 3$ принадлежит пространству $\widetilde{C}_{q-1}^{q}$. 
Доказательство. Так как $\left(\widetilde{C}_{k}^{q}, \widetilde{d}\right) \cong\left(C_{k}^{q}(X), d\right)$, доказательство можно провести для комплексов $\left(C_{k}^{q}(X), d\right)$. Обозначим через $\operatorname{supp} \xi$ носитель векторного поля $\xi$. Тогда коцепь $L \in C_{q-1}^{q}(X)$, если из того, что $L\left(\xi_{1}, \ldots, \xi_{q}\right) \neq 0$ следует, что не пусто хотя бы одно из попарных пересечений множеств $\operatorname{supp} \xi_{1}, \ldots, \operatorname{supp} \xi_{q}$.

Необходимо установить, что если $d L \in C_{q-1}^{q+1}(X)$, то для любых $\xi_{1}, \ldots, \xi_{q} \in \mathscr{U}(X)$ таких, что их носители попарно не пересекаются, справедливо равенство $L\left(\xi_{1}, \ldots, \xi_{q}\right)=0$.

При $q \geq 3$, используя разбиение единицы, инвариантное относительно действия группы, можно свести доказательство к случаю, когда носитель хотя бы одного из векторных полей $\xi_{1}, \ldots, \xi_{q}$ будет иметь окрестность диффеоморфную $\mathbb{R}$.

Тогда можно воспользоваться утверждением о том, что каждое финитное (имеющее компактный носитель) гладкое векторное поле на $\mathbb{R}^{n}$ можно предствить в виде конечной суммы $\sum_{k}\left[\eta_{k}, \zeta_{k}\right]$, где $\eta_{k}$ и $\zeta_{k}$ - гладкие финитные векторные поля на $\mathbb{R}^{n}$ (доказательство см. в [2]).

Предположим для определенности, что $\xi_{1}=\sum_{k}\left[\eta_{k}, \zeta_{k}\right]$. Так как среди множеств $\operatorname{supp} \eta_{k}, \operatorname{supp} \zeta_{k}$, $\operatorname{supp} \xi_{2}, \ldots, \operatorname{supp} \xi_{q}$ пересекаются только $\operatorname{supp} \eta_{k}$ и $\operatorname{supp} \zeta_{k}$, то

$$
\sum_{k} d L\left(\eta_{k}, \zeta_{k}, \xi_{2}, \ldots, \xi_{q}\right)=\sum_{k} L\left(\left[\eta_{k}, \zeta_{k}\right], \xi_{2}, \ldots, \xi_{q}\right)=L\left(\sum_{k}\left[\eta_{k}, \zeta_{k}\right], \xi_{2}, \ldots, \xi_{q}\right)=L\left(\xi_{1}, \ldots, \xi_{q}\right) .
$$

Так как $d L \in C_{q-1}^{q+1}(X)$, то $d L\left(\eta_{k}, \zeta_{k}, \xi_{2}, \ldots, \xi_{q}\right)=0$ для всех $k$. В противном случае среди множеств $\operatorname{supp} \eta_{k}, \operatorname{supp} \zeta_{k}, \operatorname{supp} \xi_{2}, \ldots, \operatorname{supp} \xi_{q}$ должны были быть, по крайней мере, две пары пересекающихся. Отсюда получаем необходимое равенство $L\left(\xi_{1}, \ldots, \xi_{q}\right)=0$.

Фильтрация $0=\widetilde{C}_{0}^{q} \subset \widetilde{C}_{1}^{q} \subset \ldots \subset \widetilde{C}_{q}^{q}=\mathrm{i}\left(C^{q}(\mathscr{U}(X))\right)$ порождает спектральную последовательность $\left(\widetilde{E}_{r}^{k, q-k}, \delta_{r}\right)$, нулевой член которой $\widetilde{E}_{0}^{k, q-k}$ по определению равен $\widetilde{C}_{k}^{q} / \widetilde{C}_{k-1}^{q}$. В следующем параграфе мы опишем фильтрацию пространств $\widetilde{E}_{0}^{k, q-k}$.

3. ФИЛЬТРАЦИЯ ПРОСТРАНСТВ $\widetilde{E}_{0}^{k, q-k}$.

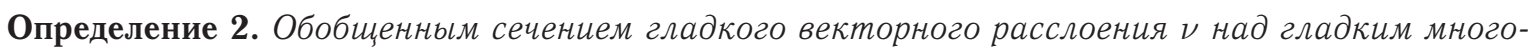
образием $M$ называется непрерывный линейный функционал на пространстве финитных (имеющих компактный носитель) гладких сечений расслоения $\nu^{\prime}$ сопряженного с $\nu$.

Обобщенное сечение расслоения $\tau_{q}^{\prime}$ называется кососимметрическим, если оно меняет знак при отображении, индуцированном перестановкой сомножителей в произведении $M \times \ldots \times M$. Пусть $F$ - обобщенное сечение некоторого расслоения $\nu$ с базой $M$. F называется сосредоточенным на замкнутом множестве $A \subset M$, если $F(\sigma)=0$ для любого сечения $\sigma$ расслоения $\nu^{\prime}$, совпадающего с нулевым сечением в окрестности множества $A$.

Для любого обобщенного сечения $F$ расслоения $\nu$, сосредоточенного на компактном множестве $A$, найдется такое число $s$, что если финитное гладкое сечение расслоения $\nu^{\prime}$ имеет в каждой точке множества $A$ касание порядка $\geq s$ с нулевым сечением, то $F(\sigma)=0$. Это утверждение следует из аналогичного утверждения для обобщенных функций [3]. Наименьшее из таких чисел $s$ называется порядком обобщенного сечения относительно множества $A$.

Пространство коцепей $C^{q}(\mathscr{U}(M))$ с помощью теоремы о ядре $[4, \S 1]$ отождествляется с пространством кососимметрических обобщенных сечений расслоения $\tau_{q}^{\prime}(M)=\pi_{1}^{*} \tau^{\prime}(M) \oplus \ldots \oplus \pi_{q}^{*} \tau^{\prime}(M)$ с базой $M^{q}=M \times \ldots \times M$, где $\pi_{i}: M^{q} \rightarrow M-$ проекция на $i$-й сомножитель, а $\pi_{i}^{*} \tau^{\prime}(M)-$ обратный образ кокасательного расслоения над $M$.

Обозначим через $M_{k}^{q}$ подмножество произведения $M^{q}$, состоящее из таких точек $\left(x_{1}, \ldots, x_{q}\right)$, что среди точек $x_{1}, \ldots, x_{q} \in M$ не более $k$ различных. Пространство $C_{k}^{q}(M)$ можно отождествить с пространством обобщенных кососимметрических сечений расслоения $\tau_{q}^{\prime}$, сосредоточенных на множестве $M_{k}^{q}$.

Обозначим через $C_{k, p}^{q}(M)$ подпространство пространства $C_{k}^{q}(M)$, состоящее из обобщенных сечений, имеющих относительно $M_{k}^{q}$ порядок $\leq q-p$. Тогда

$$
0=C_{k, q+1}^{q}(M) \subset C_{k, q}^{q}(M) \subset \ldots \subset C_{k}^{q}(M), \quad \bigcup_{p} C_{k, p}^{q}(M)=C_{k}^{q}(M) .
$$


Таким образом, подпространства $C_{k, p}^{q}(M)$ составляют фильтрацию пространства $C_{k}^{q}(M)$. Так как $d\left(C_{k, p}^{q}(M)\right) \subset C_{k, p}^{q+1}(M)$, гомоморфизм $d$ индуцирует гоморфизмы

$$
d_{k}: C_{k}^{q}(M) / C_{k-1}^{q}(M) \rightarrow C_{k}^{q+1}(M) / C_{k-1}^{q+1}(M)
$$

согласованные с фильтрациями пространств $C_{k}^{q}(M) / C_{k-1}^{q}(M)$ подпространствами

$$
C_{k, p}^{q}(M) /\left(C_{k, p}^{q}(M) \bigcap C_{k-1}^{q}(M)\right)
$$

Обозначим соответствующие спектральные последовательности через $\left(E_{k, r}^{q-p, p}(M), \delta_{k, r}\right)$.

Пусть теперь $M=S^{1}$. Фильтрация пространств $C_{k}^{q}\left(S^{1}\right)$ подпространствами $C_{k, p}^{q}\left(S^{1}\right)$ индуцирует фильтрацию пространств $\widetilde{C}_{k}^{q}$ подпространствами $\widetilde{C}_{k, p}^{q}=C_{k, p}^{q}\left(S^{1}\right) \bigcap \widetilde{C}_{k}^{q}$. Так как $d\left(C_{k, p}^{q}\left(S^{1}\right)\right) \subset$ $\subset C_{k, p}^{q+1}\left(S^{1}\right)$ и отображение і $\circ \mathrm{p}: C^{q}\left(\mathscr{U}\left(S^{1}\right)\right) \rightarrow C^{q}\left(\mathscr{U}\left(S^{1}\right)\right)$ не увеличивает порядка обобщенного сечения, то $\widetilde{d}\left(\widetilde{C}_{k, p}^{q}\right) \subset \widetilde{C}_{k, p}^{q+1}$. Таким образом, гомоморфизм $\widetilde{d}$ индуцирует гомоморфизмы

$$
\widetilde{d}_{k}: \widetilde{C}_{k}^{q} / \widetilde{C}_{k-1}^{q} \rightarrow \widetilde{C}_{k}^{q+1} / \widetilde{C}_{k-1}^{q+1}
$$

согласованные с фильтрациями пространств $\widetilde{C}_{k}^{q} / \widetilde{C}_{k-1}^{q}$ подпространствами $\widetilde{C}_{k, p}^{q} /\left(\widetilde{C}_{k, p}^{q} \cap \widetilde{C}_{k-1}^{q}\right)$. Обозначим соответствующие спектральные последовательности через $\left(\widetilde{E}_{k, r}^{q-p, p}, \delta_{k, r}\right)$.

Заметим, что фильтрация пространств $C_{k}^{q}(M)$ подпространствами $C_{k, p}^{q}(M)$ бессодержательна при $q=k$. Соответственно бессодержательна и фильтрация пространств $\widetilde{C}_{k}^{q}$ подпространствами $\widetilde{C}_{k, p}^{q}$ при $q=k$. В [2] показано, что этот случай можно не рассматривать для $M$ (и для $M=S^{1}$, в частности). Из леммы 2 следует, что мы можем не рассматривать случая $q=k$ для комплекса $\left(\widetilde{C}_{k}^{q}, \widetilde{d}\right)$ при $q \geq 3$. Одномерные и двумерные когомологии комплекса $\left(C^{*}(\mathscr{U}(X)), d\right)$ вычислены в [1] и приведены в теореме 1 , поэтому случай $q=k=1,2$ для комплекса $\left(\widetilde{C}_{k}^{q}, \widetilde{d}\right)$ также можно не рассматривать.

\section{4. ДИАГОНАЛЬНЫЙ КОМПЛЕКС И ЕГО КОГОМОЛОГИИ}

Рассмотрим сначала спектральные последовательности для диагональных комплексов $\left(\widetilde{E}_{1, r}^{q-p, p}, \delta_{1, r}\right)$ и $\left(E_{1, r}^{q-p, p}\left(S^{1}\right), \delta_{1, r}\right)$.

Согласно определению $E_{1,0}^{q-p, p}\left(S^{1}\right)=C_{1, p}^{q}\left(S^{1}\right) / C_{1, p+1}^{q}\left(S^{1}\right), \widetilde{E}_{1,0}^{q-p, p}=\widetilde{C}_{1, p}^{q} / \widetilde{C}_{1, p+1}^{q} . \widetilde{E}_{1,0}^{q-p, p}-$ подпространство в пространстве $E_{1,0}^{q-p, q}\left(S^{1}\right)$. Обозначим $q-p$ через $m$ и будем далее вместо $E_{1,0}^{q-p, p}\left(S^{1}\right)$ писать $E_{1,0}^{m, q-m}\left(S^{1}\right)$, а вместо $\widetilde{E}_{1,0}^{q-p, p}$ писать $\widetilde{E}_{1,0}^{m, q-m}$. Сформулируем основной результат параграфа.

Теорема 4. $H^{1}\left(C_{\triangle}^{*}(X)\right) \cong \mathbb{R} \oplus \mathbb{R}, H^{2}\left(C_{\triangle}^{*}(X)\right) \cong \mathbb{R} u H^{q}\left(C_{\triangle}^{*}(X)\right)=0$, при $q \geq 3$, где одномерные образующие - классы коциклов $\alpha_{1}$ и $\alpha_{2}$, а двумерная образующая класс коцикла $\beta$. Коцикль $\alpha_{1}$, $\alpha_{2}$ и $\beta$ определяются равенствами (2) и (3).

Так как $\left(C_{\triangle}^{*}(X), d\right) \cong\left(\widetilde{C}_{\triangle}^{*}(X), \widetilde{d}\right)$, мы будем рассматривать комплекс $\left(\widetilde{C}_{\triangle}^{*}, \widetilde{d}\right)$. Пространство $\widetilde{C}_{\triangle}^{q}-$ подпространство пространства $C_{\triangle}^{q}\left(S^{1}\right)$, поэтому коцепи из $\widetilde{C}_{\triangle}^{q}$ можно представлять как обобщенные сечения расслоения $\tau_{q}^{\prime}\left(S^{1}\right)$.

В нашем случае, в отличие от [2], присутствуют особые точки. Поэтому выделим в комплексе $\left(\widetilde{C}_{\triangle}^{*}, \widetilde{d}\right)$ подкомплекс $\left(\left(\widetilde{C}_{0, \pi}^{*}\right) \triangle, \widetilde{d}\right)$ коцепей, сосредоточенных в особых точках. Обозначим факторкомплекс $\widetilde{C}_{\triangle}^{*} /\left(\widetilde{C}_{0, \pi}^{*}\right) \triangle$ через $\bar{C}_{\triangle}^{*}$. Рассмотрим точную последовательность комплексов

$$
0 \rightarrow\left(\widetilde{C}_{0, \pi}^{*}\right)_{\triangle} \rightarrow \widetilde{C}_{\triangle}^{*} \rightarrow \bar{C}^{*} \rightarrow 0
$$

и соответствующую длинную точную последовательность в когомологиях

$$
\ldots \rightarrow H^{i}\left(\left(\widetilde{C}_{0, \pi}^{*}\right)_{\triangle}\right) \rightarrow H^{i}\left(\widetilde{C}_{\triangle}^{*}\right) \rightarrow H^{i}\left(\bar{C}^{*}\right) \rightarrow H^{i+1}\left(\left(\widetilde{C}_{0, \pi}^{*}\right)_{\triangle}\right) \rightarrow H^{i+1}\left(\widetilde{C}_{\triangle}^{*}\right) \rightarrow \ldots
$$

Для доказательства теоремы 4 вычислим члены $H^{i}\left(\left(\widetilde{C}_{0, \pi}^{*}\right) \triangle\right)$ и $H^{i}\left(\bar{C}^{*}\right)$ длинной точной последовательности (5). Начнем с вычисления $H^{i}\left(\left(\widetilde{C}_{0, \pi}^{*}\right) \triangle\right)$. 


\section{1. Когомологии комплекса $\left(\widetilde{C}_{0, \pi}^{*}\right) \triangle$}

Пусть $\lambda=\sum_{k_{1}, \ldots, k_{q}} c_{k_{1}, \ldots, k_{q}} y_{1}^{k_{1}} \cdot \ldots \cdot y_{q}^{k_{q}}-$ некоторый кососимметрический многочлен, тогда $\lambda$ определяет дифференциальный оператор:

$$
\lambda\left(\xi_{1}, \ldots, \xi_{q}\right)(t)=\left(\sum_{k_{1}, \ldots, k_{q}} c_{k_{1}, \ldots, k_{q}} \frac{d^{k_{1}}\left(\xi_{1}\right)}{(d t)^{k_{1}}}(t) \cdot \ldots \cdot \frac{d^{k_{q}}\left(\xi_{q}\right)}{(d t)^{k_{q}}}(t)\right) .
$$

Пусть $\varphi-$ обобщенная функция на окружности. Тогда можно рассмотреть коцепи вида $\varphi \lambda$, значение которых на векторных полях $\xi_{1}, \ldots, \xi_{q}$ определяется следующим образом:

$$
(\varphi \lambda)\left(\xi_{1}, \ldots, \xi_{q}\right)=\varphi\left(\lambda\left(\xi_{1}, \ldots, \xi_{q}\right)(t)\right) .
$$

Из теории обобщенных функций $[5, \S 4]$ следует, что всякую коцепь, сосредоточенную в особых точках, можно представить в виде линейной комбинации коцепей вида $\varphi \lambda$, где в качестве обобщенной функции $\varphi$ нужно взять либо $\delta(0)$, либо $\delta(\pi)$.

Теорема 5. $H^{1}\left(\left(\widetilde{C}_{0, \pi}^{*}\right)_{\triangle}\right) \cong \mathbb{R} \oplus \mathbb{R}, H^{q}\left(\left(\widetilde{C}_{0, \pi}^{*}\right)_{\triangle}\right)=0$ nри $q \geq 1$, где одномерные образующие классы коциклов $\alpha_{1}$ и $\alpha_{2}$, определенных равенствами (2).

Доказательство. Подкомплекс $\left(\widetilde{C}_{0, \pi}^{*}\right)_{\triangle}$ изоморфен прямой сумме двух подкомплексов $\widetilde{C}_{0}^{*}$ и $\widetilde{C}_{\pi}^{*}$ коцепей, сосредоточенных в точке 0 и в точке $\pi$.

Вычислим когомологии комплекса $\widetilde{C}_{0}^{*}$. Обозначим через $\alpha_{n}^{0}$ коцепь вида $\delta(0) y^{n}$. Всякую коцепь $L \in \widetilde{C}_{0}^{q}$ можно представить в виде

$$
L=\sum_{n_{1}, \ldots, n_{q}} c_{n_{1}, \ldots, n_{q}} \alpha_{n_{1}}^{0} \wedge \ldots \wedge \alpha_{n_{q}}^{0},
$$

где сумма имеет конечное число слагаемых $c_{n_{1}, \ldots, n_{q}}-$ константы, а все числа $n_{1}, \ldots, n_{q}-$ нечетные.

По определению дифференциала $\widetilde{d}$

$$
\widetilde{d}\left(\alpha_{n_{1}}^{0} \wedge \ldots \wedge \alpha_{n_{q}}^{0}\right)=\sum_{i=1}^{q}(-1)^{i-1} \sum_{\substack{j=1, j-\text { нечетно }}}^{\left[n_{i} / 2\right]}\left(C_{n_{i}}^{j}-C_{n_{i}}^{n_{i}-j+1}\right) \alpha_{j}^{0} \wedge \alpha_{n_{i}-j+1}^{0} \wedge \alpha_{n_{1}}^{0} \wedge \ldots \wedge \widehat{\alpha_{n_{i}}^{0}} \wedge \ldots \wedge \alpha_{n_{q}}^{0} .
$$

Представим коцепь $L \in \widetilde{C}_{0}^{q}$ в виде

$$
L=\sum_{n_{1}, \ldots, n_{q} \neq 1} c_{n_{1}, \ldots, n_{q}}^{1} \alpha_{n_{1}}^{0} \wedge \ldots \wedge \alpha_{n_{q}}^{0}+\sum_{l_{1}, \ldots, l_{q-1}} c_{l_{1}, \ldots, l_{q}}^{2} \alpha_{1}^{0} \wedge \alpha_{l_{1}}^{0} \wedge \ldots \wedge \alpha_{l_{q-1}}^{0}
$$

Положим $n=\sum_{i=1}^{q} n_{i}, l=\sum_{i=1}^{q-1} l_{i}$. Пусть $q>1$, рассмотрим оператор $F^{q}: \widetilde{C}_{0}^{q} \rightarrow \widetilde{C}_{0}^{q-1}$ следующего вида:

$$
F^{q} L=\sum_{l_{1}, \ldots, l_{q-1} \neq 1} \frac{c_{l_{1}, \ldots, l_{q-1}}^{2}}{l+1-q} \alpha_{l_{1}}^{0} \wedge \ldots \wedge \alpha_{l_{q-1}}^{0}
$$

Прямыми вычислениями можно показать, что справедливо равенство

$$
F^{q+1} \circ \widetilde{d}+\widetilde{d} \circ F^{q}=i d_{\widetilde{C}_{0}^{q}},
$$

то есть $F$ - оператор гомотопии. Следовательно, $H^{k}\left(\widetilde{C}_{0}^{*}\right)=0$, при $k>1$.

Рассмотрим случай $q=1$. Одномерным коциклом является только $\alpha_{1}^{0}=\alpha_{1}$, класс которого и будет образующей в $H^{1}\left(\widetilde{C}_{0}^{*}\right)$.

Когомологии комплекса $\widetilde{C}_{\pi}^{*}$ вычисляются аналогично. $H^{k}\left(\widetilde{C}_{\pi}^{*}\right)=0$, при $k \geq 1$ и $H^{1}\left(\widetilde{C}_{\pi}^{*}\right) \cong \mathbb{R}$, где одномерная образующая - класс коцикла $\alpha_{2}$. 


\section{2. Когомологии фрактор-комплекса $\bar{C}_{\triangle}^{*}$}

Напомним некоторые факты из [2], которые будут необходимы в дальнейшем. Всякий элемент из $E_{1,0}^{m, q-m}\left(S^{1}\right)$ можно представить в виде суммы $\sum_{i} \varphi_{i} \lambda_{i}$, где $\lambda_{i}-$ элемент фактор-пространства $P_{m}^{q}=\widetilde{P}_{m}^{q} / R_{m}^{q}$ пространства кососимметрических однородных многочленов степени $m$ от $q$ переменных $\widetilde{P}_{m}^{q}$ по идеалу $R_{m}^{q}$, порожденному многочленом $\sigma_{q}=y_{1}+\ldots+y_{q}$, а $\varphi_{i}-$ обобщенные функции на окружности. Будем элементы фактор-пространства $P_{m}^{q}$ и представляющие их многочлены обозначать одинаковыми символами. Значение коцепи $\varphi \lambda$ определяется формулами (6) и (7).

Рассмотрим отображение $\widetilde{\nabla}: \widetilde{P}_{m}^{q} \rightarrow \widetilde{P}_{m+1}^{q+1}$, которое определяется следующим образом:

$$
\widetilde{\nabla}(\lambda)\left(y_{1}, \ldots, y_{q+1}\right)=\sum_{1 \leq i<j \leq q+1}(-1)^{i+j-1}\left(y_{j}-y_{i}\right) \lambda\left(y_{i}+y_{j}, y_{1}, \ldots, \widehat{y}_{i}, \ldots, \widehat{y_{j}}, \ldots, y_{q+1}\right) .
$$

Из определения дифференциала (1) следует равенство

$$
d(\varphi \lambda)=\varphi \widetilde{\nabla}(\lambda) .
$$

Так как $\widetilde{\nabla}\left(R_{m}^{q}\right) \subset R_{m+1}^{q+1}$, то $\widetilde{\nabla}$ индуцирует отображение $\nabla: P_{m}^{q} \rightarrow P_{m+1}^{q+1}$.

Из определения дифференцирования $[3,5]$ обобщенных функций следует равенство

$$
\varphi \cdot\left(\sigma_{q} \lambda\right)=-\frac{d \varphi}{d t} \lambda
$$

Тогда из равенств (8) и (9) следует, что $\delta_{0}(\varphi \lambda)=\varphi \nabla(\lambda)$, где $\delta_{0}: E_{1,0}^{m, q-m}\left(S^{1}\right) \rightarrow E_{1,0}^{m+1, q-m}\left(S^{1}\right)-$ дифференциал комплекса $E_{1,0}^{m, q-m}\left(S^{1}\right)$, индуцированный дифференциалом $d$. Образующие когомологий комплекса $\left(P_{m}^{q}, \nabla\right)$ равны классам многочленов $\lambda^{2}=y_{1}^{2} y_{2}-y_{1} y_{2}^{2}$ и $\lambda^{3}=\left(y_{1}-y_{2}\right)\left(y_{2}-y_{3}\right)\left(y_{3}-y_{1}\right)$. Таким образом, получается, что

$$
E_{1,1}^{3,-1}\left(S^{1}\right)=\varphi \widetilde{\lambda}_{2}, \quad E_{1,1}^{3,0}\left(S^{1}\right)=\psi \widetilde{\lambda}_{3}, \quad E_{1,1}^{m, q-m}\left(S^{1}\right)=0, \quad \text { если } \quad m \neq 3, q-m \neq 0,1 .
$$

Перейдем к нашему случаю. Пусть ј: $\widetilde{C}_{\triangle}^{q} \rightarrow \bar{C}^{q}$ проекция на фактор-комплекс, $\bar{d}-$ дифференциал фактор-комплекса $\bar{C}^{*}$, индуцированный дифференциалом $\widetilde{d}$. Обозначим через $\bar{C}_{p}^{q}=\mathrm{j}\left(\widetilde{C}_{1, p}^{q}\right)$. Тогда $\bar{C}_{p}^{q} \subset \bar{C}_{p-1}^{q}$ и $\bar{d}\left(\bar{C}_{p}^{q}\right) \subset \bar{C}_{p}^{q+1}$. Поэтому пространства $\bar{C}_{p}^{q}$ задают фильтрацию пространства $\bar{C}^{q}$, согласованную с дифференциалом $\bar{d}$. Обозначим соответствующую спектральную последовательность через $\bar{E}_{1,0}^{m, q-m}$, где как и ранее $m=q-p$. Согласно определению нулевого члена спектральной последовательности

$$
\bar{E}_{1,0}^{m, q-m}=\bar{C}_{q-m}^{q} / \bar{C}_{q-m+1}^{q}=\mathrm{j}\left(\widetilde{C}_{1, q-m}^{q}\right) / \mathrm{j}\left(\widetilde{C}_{1, q-m+1}^{q}\right)=\widetilde{C}_{1, q-m}^{q} /\left(\widetilde{C}_{1, q-m+1}^{q}+\widetilde{C}_{1, q-m}^{q} \bigcap\left(\widetilde{C}_{0, \pi}^{*}\right)_{\triangle}\right) .
$$

Проекция ј индуцирует сюръективное отображение $\mathrm{J}: \widetilde{E}_{1,0}^{m, q-m} \rightarrow \bar{E}_{1,0}^{m, q-m}$, которое классу коцепи в $\widetilde{E}_{1,0}^{m, q-m}=\widetilde{C}_{1, q-m}^{q} / \widetilde{C}_{1, q-m+1}^{q}$ ставит в соответствие ее класс в $\widetilde{C}_{1, q-m}^{q} /\left(\widetilde{C}_{1, q-m+1}^{q}+\widetilde{C}_{1, q-m}^{q} \bigcap\left(\widetilde{C}_{0, \pi}^{*}\right)_{\triangle}\right)$. Дифференциал в комплексе $\bar{E}_{1,0}^{m, q-m}$, индуцированный дифференциалом $\bar{d}$, обозначим через $\bar{\delta}_{0}$.

Назовем обобщенную функцию $\varphi$ на окружности нечетной (четной), если ее значение на четных(нечетных) функциях равно нулю. Любую обобщенную функцию можно однозначно разложить в сумму нечетной и четной обобщенной функции. Справедлива следующая лемма.

Лемма 3.

$$
\begin{aligned}
& \bar{E}_{1,1}^{3,-1}=\varphi \widetilde{\lambda}_{2}, \\
& \bar{E}_{1,1}^{3,0}=\psi \widetilde{\lambda}_{3}, \\
& \bar{E}_{1,1}^{m, q-m}=0, \text { если } \quad m \neq 3, q-m \neq 0,1,
\end{aligned}
$$

где $\varphi$ - нечетная обобщенная функция, $\psi$ - четная обобщенная функция, $\varphi$ и $\psi$ заданы с точностью до обобщенных функций, сосредоточенных в особых точках. Дифференциал $\bar{\delta}_{1}: \bar{E}_{1,1}^{3,-1} \rightarrow \bar{E}_{1,1}^{3,0}$ индуцирован дифференцилом $\widetilde{d}$, значение которого на коцепи $\widetilde{\lambda}^{2}$ определяется $\kappa a \kappa \widetilde{d}\left(\varphi \widetilde{\lambda}^{2}\right)=-\frac{d \varphi}{d t} \widetilde{\lambda}^{3}$. 
Доказательство. Образ коцепи $\varphi \lambda$ при отображении р: $C^{q}\left(\mathscr{U}\left(S^{1}\right)\right) \rightarrow C^{q}(\mathscr{U}(X))$ будем также обозначать через $\varphi \lambda$. Рассмотрим отображение і о p: $C^{q}\left(\mathscr{U}\left(S^{1}\right)\right) \rightarrow \mathrm{i}\left(C^{q}(\mathscr{U}(X))\right) \subset C^{q}\left(\mathscr{U}\left(S^{1}\right)\right)$. Это

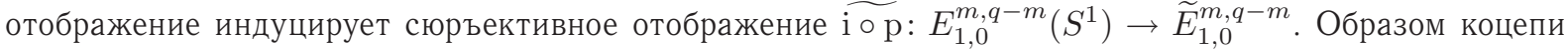
$\varphi \lambda$ при отображении і ор будет коцепь $\varphi \widetilde{\lambda}$, значение которой на векторных полях $\xi_{1}, \ldots, \xi_{q}$ определяется следующим образом:

$$
\begin{gathered}
(\varphi \tilde{\lambda})\left(\xi_{1}, \ldots, \xi_{q}\right)=\varphi\left(\widetilde{\lambda}\left(\xi_{1}, \ldots, \xi_{q}\right)(t)\right), \quad \text { где } \quad \tilde{\lambda}\left(\xi_{1}, \ldots, \xi_{q}\right)(t)= \\
=\frac{1}{2^{q}}\left(\sum_{k_{1}+\ldots+k_{q}=m} c_{k_{1}, \ldots, k_{q}} \frac{d^{k_{1}}\left(\xi_{1}(t)-\xi_{1}(-t)\right)}{(d t)^{k_{1}}}(t) \cdot \ldots \cdot \frac{d^{k_{q}}\left(\xi_{q}(t)-\xi_{q}(-t)\right)}{(d t)^{k_{q}}}(t)\right) .
\end{gathered}
$$

Это следует из того, что $\varphi \widetilde{\lambda}\left(\xi_{1}, \ldots, \xi_{q}\right)=0$, если среди аргументов есть четное векторное поле и $\varphi \widetilde{\lambda}\left(\xi_{1}, \ldots, \xi_{q}\right)=\varphi \lambda\left(\xi_{1}, \ldots, \xi_{q}\right)$, если все аргументы нечетные. Тогда $\mathrm{i}(\varphi \lambda)=\varphi \widetilde{\lambda}$ и $\mathrm{p}(\varphi \widetilde{\lambda})=\varphi \lambda$, где $\varphi \lambda$ рассматривается как элемент пространства $C^{q}(\mathscr{U}(X))$. Заметим, что функция $\widetilde{\lambda}\left(\xi_{1}, \ldots, \xi_{q}\right)(t)$ будет нечетной (четной) при любых $\xi_{1}, \ldots, \xi_{q} \in \mathscr{U}\left(S^{1}\right)$, если $\lambda \in \widetilde{P}_{m}^{q}$, где разность $m-q$ нечетное (четное) целое число. Тогда коцепи вида $\varphi \widetilde{\lambda}$ будут равны нулю, если $\varphi-$ нечетная (четная) обобщенная функция, а $\lambda \in \widetilde{P}_{m}^{q}$, где $m-q-$ четное (нечетное) число. Поэтому можно считать, что в представлении элемента из $\widetilde{E}_{1,0}^{m, q-m}$ в виде $\sum_{i} \varphi_{i} \widetilde{\lambda}_{i}$ все обобщенные функции $\varphi_{i}$ четные (нечетные), если $m-q$ четно (нечетно).

Так как любой элемент пространства $\widetilde{E}_{1,0}^{m, q-m}$ можно представить в виде $\sum_{i} \varphi_{i} \widetilde{\lambda}_{i}$, то элементы пространства $\bar{E}_{1,0}^{m, q-m}$ можно представлять в виде $\sum_{i} \varphi_{i} \widetilde{\lambda}_{i}$, где обобщенные функции $\varphi_{i}$ заданы с точностью до обобщенных функций, сосредоточенных в особых точках, и их четность зависит от степени многочлена $\lambda_{i}$.

Из равенства (8) и определения дифференциала $\widetilde{d}$ следует, что

$$
\widetilde{d}(\varphi \widetilde{\lambda})=\mathrm{i} \circ \mathrm{p}(d \varphi \widetilde{\lambda})=\mathrm{i}(d(\mathrm{p}(\varphi \widetilde{\lambda}))=\mathrm{i}(d \varphi \lambda)=\mathrm{i}(\varphi \widetilde{\nabla} \lambda)=\varphi \widetilde{\nabla} \lambda .
$$

Из равенства (9) следует равенство

$$
\varphi \cdot\left(\widetilde{\sigma_{q} \lambda}\right)=-\frac{d \varphi}{d t} \widetilde{\lambda}
$$

Тогда получаем, что $\bar{\delta}_{0}(\varphi \widetilde{\lambda})=\varphi \widetilde{\nabla \lambda}$, и действие дифференциала $\overline{\delta_{0}}$ на элементах из $\bar{E}_{1,0}^{m, q-m}$ сводится к действию дифференциала $\nabla$ на классах многочленов из пространства $P_{m}^{q}$, откуда следует утверждение леммы.

Для вычисления члена $\bar{E}_{1,2}^{m, q-m}$ нам понадобятся некоторые сведения из теории потоков де Рама [6].

Определение 3. Потоком де Рама степени q на многообразии $M$ размерности $n$ называется непрерывный линейный функционал на пространстве дифференциальных форм степени $n-q$ с компактными носителями. Дифференциал $b: D^{q}(M) \rightarrow D^{q+1}(M)$ определяется формулой $b K(\omega)=K(d \omega)$, где $K-$ поток степени $q, \omega-$ дифференциальная форма степени $n-q-1$.

Рассмотрим комплекс потоков де Рама на окружности $\left(D^{q}\left(S^{1}\right), b\right)$. Всякий поток степени 0 на окружности можно представить в виде $\varphi$, а поток степени 1 - в виде $\psi d t$, где $\varphi, \psi-$ обобщенные функции на окружности. Значения потоков $\varphi$ и $\psi d t$ на дифференциальных формах определяются следующим образом:

$$
\varphi(g d t)=\varphi(g), \quad \psi d t(f)=\psi(f),
$$

где $g$ и $f$ - гладкие периодические функции на $\mathbb{R}$. Формулы для дифференциала $b$ в этом случае перепишутся в виде

$$
b(\psi d t)=0, \quad(b \varphi)(f)=\varphi(d f)=\varphi\left(\frac{d f}{d t} d t\right)=-\frac{d \varphi}{d t} d t(f) .
$$

Под потоками на орбифолде будем понимать потоки на окружности, инвариантные относительно действия группы $\mathbb{Z}_{2}$ или, что равносильно, непрерывные линейные функционалы на пространстве 
дифференциальных форм на орбифолде (дифференциальные формы на орбифолде - дифференциальные формы на окружности, инвариантные относительно действия группы $\mathbb{Z}_{2}$ ). Таким образом, комплекс потоков на орбифолде - подкомплекс комплекса потоков на окружности. Обозначим его через $\left(D^{q}(X), b\right)$.

0-формами на орбифолде являются гладкие, четные периодические функции на $\mathbb{R}, 1$-формами на орбифолде являются выражения вида $g d t$, где $g$ - гладкая нечетная периодическая функция на $\mathbb{R}$.

Поток степени 0 на орбифолде можно представить в виде $\varphi$, а поток степени 1 - в виде $\psi d t$, где $\varphi-$ нечетная обобщенная функция на окружности, $\psi-$ четная обобщенная функция на окружности. Дифференциал определяется так же как и для комплекса потоков на окружности.

Обозначим через $\left(D_{A}^{q}(X), b\right)$ подкомплекс комплекса $\left(D^{q}(X), b\right)$, состоящий из потоков, сосредоточенных на множестве $A=\{0, \pi\}$. В терминах обобщенных функций это означает, что обобщенные функции $\varphi$ и $\phi$ сосредоточены в особых точках. Обозначим через $\bar{D}^{q}=D^{q}(X) / D_{A}^{q}(X)$ факторкомплекс комплекса потоков на орбифолде по подкомплексу потоков, сосредоточенных в особых точKax.

Сопоставляя коцепи $\varphi \widetilde{\lambda}^{2}$ поток $\varphi$, коцепи $\psi \widetilde{\lambda}^{3}$ поток $\psi d t$, определим отображение $\gamma: \bar{E}_{1,1}^{3, q-1} \rightarrow \bar{D}^{q}$. При этом дифференциал комплекса $\bar{E}_{1,1}^{3, q-1}$ перейдет в дифференциал комплекса $\bar{D}^{q}$. Так как $\gamma-$ биективное отображение, то $\gamma$ определяет изоморфизм комплексов. Найдем когомологии комплекса $\bar{D}^{q}$.

Обозначим через $\left(D_{A}^{q}\left(S^{1}\right), b\right)$ подкомплекс комплекса $\left(D^{q}\left(S^{1}\right), b\right)$, состоящий из потоков, сосредоточенных в точках 0 и $\pi$. Тогда комплекс $\left(D_{A}^{q}(X), b\right)$ - подкомплекс инвариантных потоков комплекса $\left(D_{A}^{q}\left(S^{1}\right), b\right)$. Так как множество $A$ неподвижно относительно действия группы $\mathbb{Z}_{2}$, то отсюда следует, что комплекс $\bar{D}^{q}=D^{q}(X) / D_{A}^{q}(X)$ изоморфен подкомплексу инвариантных элементов комплекса $D^{q}\left(S^{1}\right) / D_{A}^{q}\left(S^{1}\right)$. Обозначим его через $\left(D^{q}\left(S^{1}\right) / D_{A}^{q}\left(S^{1}\right)_{\text {Inv }}\right.$.

В дальнейшем нам понадобится лемма об инвариантных когомологиях комплекса, на котором действует конечная группа.

Лемма 4. Пусть $\left(B^{q}, d\right)$ - дифференциальньй комплекс, на котором автоморфизмами ком-

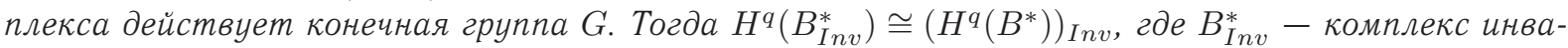
риантных коцеепей, $\left(H^{q}\left(B^{*}\right)\right)_{\text {Inv }}-$ инвариантная часть в когомологиях.

Доказательство. Пусть і: $B_{I n v}^{q} \rightarrow B^{q}-$ отображение вложения подкомплекса в комплекс. Рассмотрим также отображение $\mathrm{pr}: B^{q} \rightarrow B_{\text {Inv }}^{q}$, которое определяется формулой

$$
\operatorname{pr}(c)=\frac{1}{p} \sum_{g \in G} g c,
$$

где $c \in B^{q}, p-$ порядок группы $G$. При этом iopr $=\mathrm{id}$. Пусть i* и $\mathrm{pr}^{*}-$ индуцированные отображения в когомологиях. Тогда получаем, что $\mathrm{i}^{*} \circ \mathrm{pr}^{*}=\mathrm{id}$, откуда следует, что $\mathrm{i}^{*}-$ инъективно. Таким образом, $H^{q}\left(B_{\text {Inv }}^{*}\right) \cong \operatorname{Im~i}^{*}\left(H^{q}\left(B^{*}\right)\right.$. В свою очередь, $\mathrm{i}^{*}\left(H^{q}\left(B^{*}\right) \cong\left(H^{q}\left(B^{*}\right)\right)_{\text {Inv }}\right.$, что завершает доказательство леммы.

Применяя лемму 4 к комплексу $D^{*}\left(S^{1}\right) / D_{A}^{*}\left(S^{1}\right)$ и группе $\mathbb{Z}_{2}$, получаем что

$$
H^{q}\left(\left(D^{*}\left(S^{1}\right) / D_{A}^{*}\left(S^{1}\right)\right)_{\text {Inv }}\right) \cong\left(H^{q}\left(D^{*}\left(S^{1}\right) / D_{A}^{*}\left(S^{1}\right)\right)_{\text {Inv }} .\right.
$$

Лемма 5. Пусть $M-$ компактное ориентируемое $n$-мерное гладкое многообразие, $A-$ его замкнутое подмножество такое, что $X \backslash A-$ гладкое многообразие. Обозначим через $D^{*}(M)$ комплекс потоков на $M$ и через $D_{A}^{*}(M)$ комплекс потоков на $M$, сосредоточенных на $A$. Тогда $H^{k}\left(D^{*}(M) / D_{A}^{*}(M)\right) \cong H_{n-k}(M, A) \cong H^{k}(M \backslash A)$, где $H_{n-k}(M, A)$ - относительные гомологии, $H^{k}(M \backslash A)-$ когомологии де Рама многообразия $M \backslash A$.

Доказательство. Рассмотрим точную последовательность комплекса $D^{*}(M)$, подкомплекса $D_{A}^{*}(M)$ и соответствующую длинную точную последовательность в когомологиях

$$
\begin{gathered}
\ldots \rightarrow H^{i}\left(D_{A}^{*}(M)\right) \rightarrow H^{i}\left(D^{*}(M)\right) \rightarrow H^{i}\left(D^{*}(M) / D_{A}^{*}(M)\right) \rightarrow H^{i+1}\left(D_{A}^{*}(M)\right) \rightarrow \\
\rightarrow H^{i+1}\left(D^{*}(M)\right) \rightarrow \ldots .
\end{gathered}
$$

Из теоремы де Рама для $M[6]$ следует, что $H^{i}\left(D^{*}(M)\right) \cong H_{n-i}(M, \mathbb{R}) \cong H^{i}(M, \mathbb{R})$, где $H_{n-i}(M, \mathbb{R})$ и $H^{i}(M, \mathbb{R})$ - сингулярные гомологии и когомологии $M$. Найдем $H^{i}\left(D_{A}^{*}(M)\right)$, используя теорию пучков. 
Обозначим через $\mathfrak{D}_{A}^{q}(M)$ и $\mathfrak{D}_{A}^{q}(M) \mid A$ пучок потоков степени $q$, сосредоточенных на $A$, с базой $M$ и индуцированный пучок с базой $A$. Так как пучок $\mathfrak{D}_{A}^{q}(M)$ сконцентрирован на $A$, то существует изоморфизм $\Gamma\left(\mathfrak{D}_{A}^{q}(M)\right) \cong \Gamma\left(\mathfrak{D}_{A}^{q}(M) \mid A\right)$, где $\Gamma\left(\mathfrak{D}_{A}^{q}(M)\right)$ и $\Gamma\left(\mathfrak{D}_{A}^{q}(M) \mid A\right)-$ сечения соответствующих пучков над своими базами ([7], лемма 4.9.2). Поэтому при вычислении $H^{k}\left(D_{A}^{*}(M)\right)$ мы можем рассматривать сечения пучка $\mathfrak{D}_{A}^{q}(M) \mid A$ над $A$ вместо сечений пучка $\mathfrak{D}_{A}^{q}(M)$ над $X$.

Пучок $\mathfrak{D}_{A}^{q}(M) \mid A$ образует резольвенту постоянного пучка над $A$, состоящую из мягких пучков. Тогда $H^{i}\left(D_{A}^{*}(M)\right) \cong H^{i}(A, \mathbb{R}) \cong H_{n-i}(A, \mathbb{R})$, где $H_{n-i}(A, \mathbb{R})$ и $H^{k}(A, \mathbb{R})-$ сингулярные гомологии и когомологии $A$ соответственно.

Поставив цепи из комплекса относительных гомологий $C^{q}(M, A)$ в соответствие поток, представляющий собой интеграл по этой цепи, получим отображение $C^{q}(M, A) \rightarrow D^{*}(M) / D_{A}^{*}(M)$, которое индуцирует соответствующее отображение в когомологиях. Тогда, используя (11), спектральную последовательность

$$
\ldots \rightarrow H_{n-i}(A, \mathbb{R}) \rightarrow H_{n-i}(M, \mathbb{R}) \rightarrow H_{n-i}(M, A) \rightarrow H_{n-i-1}(A, \mathbb{R}) \rightarrow H_{n-i-1}(M, \mathbb{R}) \rightarrow \ldots,
$$

изоморфизмы

$$
\begin{array}{ll}
H_{n-i}(A, \mathbb{R}) \cong H^{i}\left(D_{A}^{*}(M)\right), & H_{n-i-1}(A, \mathbb{R}) \cong H^{i+1}\left(D_{A}^{*}(M)\right), \\
H_{n-i}(M, \mathbb{R}) \cong H^{i}\left(D^{*}(M)\right), & H_{n-i-1}(M, \mathbb{R}) \cong H^{i+1}\left(D^{*}(M)\right)
\end{array}
$$

и лемму о пяти гомоморфизмах, получаем изоморфизм

$$
H_{n-i}(M, A) \cong H^{i}\left(D^{*}(M) / D_{A}^{*}(M)\right) .
$$

В свою очередь, $H_{n-i}(M, A) \cong H^{n}(M \backslash A)$ ([8, §17, теорема 14 и упражнение 44]), что завершает доказательство леммы.

Применяя лемму 5 в случае $M=S^{1}, A=\{0, \pi\}$, получим, что

$$
H^{q}\left(D^{*}\left(S^{1}\right) / D_{A}^{*}\left(S^{1}\right)\right) \cong H_{1-q}\left(S^{1} ; A\right) \cong H^{q}\left(S^{1} \backslash A\right) .
$$

Пространство $S^{1} \backslash A$ представляет собой несвязное объединение двух открытых интервалов, поэтому

$$
H^{0}\left(S^{1} \backslash A\right) \cong \mathbb{R} \oplus \mathbb{R}, \quad H^{q}\left(S^{1} \backslash A\right)=0 \quad \text { при } \quad q \geq 0 .
$$

Образующим в $H^{0}\left(S^{1} \backslash A\right)$ соответствуют классы потоков $\varphi_{1}=\int_{0}^{\pi} d t$ и $\varphi_{2}=\int_{\pi}^{2 \pi} d t$ в $H^{q}\left(D^{*}\left(S^{1}\right) / D_{A}^{*}\left(S^{1}\right)\right.$. Так как инвариантные части от $\varphi_{1}$ и $\varphi_{2}$ отличаются лишь знаком, получим, что $H^{0}\left(\bar{D}^{*}\right) \cong \mathbb{R}$ и $H^{q}\left(\bar{D}^{*}\right)=0$ при $q \geq 0$.

Тогда получаем $\bar{E}_{1,2}^{3,-1} \cong \mathbb{R}$ и $\bar{E}_{1,2}^{m, q-m}=0$ при $q \neq 2, m \neq 3$.

Рассмотрим коцепь $\beta$, определенную формулой (3). Она определяет образующую пространства $\bar{E}_{1,2}^{3,-1}$ и является коциклом всего комплекса $\left(\bar{C}^{*}, \bar{d}\right)$, не являющимся границей [1]. Поэтому следующие дифференциалы $\bar{\delta}_{k}=0$, при $k \geq 2$, и $\bar{E}_{1,2}^{m, q-m}=\bar{E}_{1,3}^{m, q-m}=\ldots=\bar{E}_{1, \infty}^{m, q-m}$. Получаем $H^{2}\left(\bar{C}^{*}\right) \cong \mathbb{R}$ и $H^{q}\left(\bar{C}^{*}\right)=0$, если $q \neq 2$. Таким образом мы доказали следующую теорему.

Теорема 6. $H^{2}\left(\bar{C}^{*}\right) \cong \mathbb{R} u H^{q}\left(\bar{C}^{*}\right)=0$, если $q \neq 2$.

Из длинной точной последовательности (5) для комплекса $\widetilde{C}_{\triangle}^{*}$ и подкомплекса $\left(\widetilde{C}_{0, \pi}^{*}\right) \triangle$ получим, что $H^{1}\left(\widetilde{C}_{\triangle}^{*}\right) \cong \mathbb{R} \oplus \mathbb{R}, H^{2}\left(\widetilde{C}_{\triangle}^{*}\right) \cong \mathbb{R}$ и $H^{q}\left(\widetilde{C}_{\triangle}^{*}\right)=0$ при $q \neq 1,2$. Таким образом, мы доказали теорему 4.

\section{5. КОГОМОЛОГИИ КОМПЛЕКСОВ $\widetilde{C}_{k}^{q}(X) / \widetilde{C}_{k-1}^{q}(X)$}

Сформулируем вначале основной результат параграфа.

Tеорема 7. $H^{2 k}\left(\widetilde{C}_{k}^{*}(X) / \widetilde{C}_{k-1}^{*}(X)\right) \cong \mathbb{R}, H^{2 k-1}\left(\widetilde{C}_{k}^{*}(X) / \widetilde{C}_{k-1}^{*}(X)\right) \cong \mathbb{R} \oplus \mathbb{R}$, $H^{2 k-2}\left(\widetilde{C}_{k}^{*}(X) / \widetilde{C}_{k-1}^{*}(X)\right) \cong \mathbb{R} u H^{q}\left(\widetilde{C}_{k}^{*}(X) / \widetilde{C}_{k-1}^{*}(X)\right)=0$, nрu $q \neq 2 k, 2 k-1,2 k-2$, где образу-

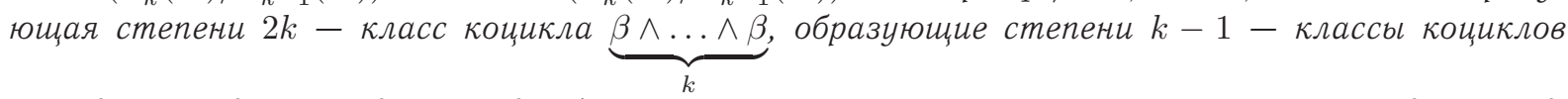

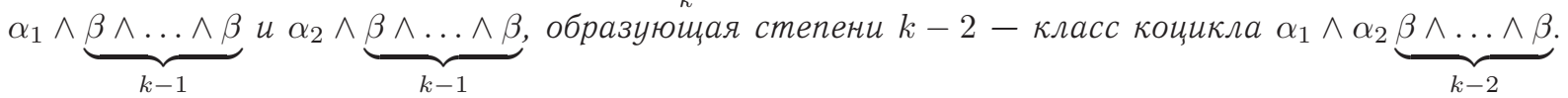
Коциклы $\alpha_{1}, \alpha_{2}$ и $\beta$ определяются равенствами (2) и (3). 
Обозначим через $\left(\widetilde{C}_{0, \pi}^{q}\right)_{k}$ подкомплекс комплекса $\widetilde{C}_{k}^{q} / \widetilde{C}_{k-1}^{q}$, состоящий из линейных комбинаций коцепей вида $L_{1} \wedge L_{2}$, где $L_{1} \in\left(\widetilde{C}_{0, \pi}^{p}\right)_{\triangle}, L_{2} \in \widetilde{C}_{k-1}^{r}, p+r=q$. Обозначим через $\bar{C}_{k}^{q}$ соответствующий фактор-комплекс.

Вычислим сначала когомологии фактор-комплекса $\bar{C}_{k}^{*}$, затем когомологии подкомплекса $\left(\widetilde{C}_{0, \pi}^{*}\right)_{k}$ и найдем когомологии всего комплекса $\widetilde{C}_{k}^{*} / \widetilde{C}_{k-1}^{*}$ из соответствующей длинной точной последовательности:

$$
\left.\cdots \rightarrow H^{i}\left(\left(\widetilde{C}_{0, \pi}^{*}\right)_{k}\right) \rightarrow H^{i}\left(\widetilde{C}_{k}^{*} / \widetilde{C}_{k-1}^{*}\right) \rightarrow H^{i}\left(\bar{C}_{k}^{*}\right) \rightarrow H^{i+1}\left(\widetilde{C}_{0, \pi}^{*}\right)_{k}\right) \rightarrow H^{i+1}\left(\widetilde{C}_{k}^{*} / \widetilde{C}_{k-1}^{*}\right) \rightarrow \ldots
$$

\section{1. Когомологии фрактор-комплекса $\bar{C}_{k}^{*}$}

Напомним некоторые результаты из [2], которые будут необходимы далее. Всякий элемент пространства $E_{k, 0}^{m, q-m}\left(S^{1}\right)$ может быть представлен в виде $\sum \varphi\left(t_{1}, \ldots, t_{k}\right)\left(\lambda_{1} \otimes \ldots \otimes \lambda_{k}\right)$, где $\varphi-$ обобщенная функция на $\left(S^{1}\right)^{k} /\left(S^{1}\right)_{k-1}^{k}, \lambda_{i} \in P_{m_{i}}^{s_{i}}, m_{1}+\ldots+m_{k}=m, s_{1}+\ldots+s_{k}=q$, а значение коцепи на векторных полях определяется следующим образом:

$$
\begin{gathered}
\sum \varphi\left(t_{1}, \ldots, t_{k}\right)\left(\lambda_{1} \otimes \ldots \otimes \lambda_{k}\right)\left(\xi_{1}, \ldots, \xi_{q}\right)=\sum\left(\sum_{\sigma \in S_{s_{1}, \ldots, s_{k}}} \varepsilon(\sigma) \varphi\left(t_{1}, \ldots, t_{k}\right) \times\right. \\
\times\left(\lambda_{1}\left(\xi_{\sigma(1)}, \ldots, \xi_{\sigma\left(s_{1}\right)}\right)\left(t_{1}\right) \cdot \ldots \cdot\left(\lambda_{k}\left(\xi_{\sigma\left(q-s_{k}\right)}, \ldots, \xi_{\sigma(q)}\right)\left(t_{k}\right)\right)\right),
\end{gathered}
$$

где $S_{s_{1}, \ldots, s_{k}}-$ такие перестановки чисел $1, \ldots, q$, что

$$
\sigma(1)<\ldots<\sigma\left(s_{1}\right), \ldots, \sigma\left(q-s_{k}\right)<\ldots<\sigma(q),
$$

$\varepsilon(\sigma)=1$, если $\sigma-$ четная перестановка, и $\varepsilon(\sigma)=-1$, если $\sigma-$ нечетная перестановка.

Коцепи $\varphi\left(t_{1}, \ldots, t_{k}\right)\left(\lambda_{1} \otimes \ldots \otimes \lambda_{k}\right)$ удовлетворяют следующему условию инвариантности:

$$
\begin{gathered}
\varphi\left(t_{1}, \ldots, t_{i}, \ldots, t_{j}, \ldots t_{k}\right)\left(\lambda_{1} \otimes \ldots \otimes \lambda_{i} \otimes \ldots \otimes \lambda_{j} \otimes \ldots \otimes \lambda_{k}\right)= \\
=(-1)^{\operatorname{deg}\left(\lambda_{i}\right) \operatorname{deg}\left(\lambda_{j}\right)} \varphi\left(t_{1}, \ldots, t_{j}, \ldots, t_{i}, \ldots t_{k}\right)\left(\lambda_{1} \otimes \ldots \otimes \lambda_{j} \otimes \ldots \otimes \lambda_{i} \otimes \ldots \otimes \lambda_{k}\right) .
\end{gathered}
$$

Дифференциал $\delta_{k, 0}$ комплекса $E_{k, 0}^{m, q-m}\left(S^{1}\right)$ действует по формуле

$$
\delta_{k, 0}\left(\varphi\left(\lambda_{1} \otimes \ldots \otimes \lambda_{k}\right)\right)=\sum_{i=1}^{k}(-1)^{s_{1}+\ldots+s_{i-1}} \varphi\left(\lambda_{1} \otimes \ldots \otimes \nabla \lambda_{i} \otimes \ldots \otimes \lambda_{k}\right),
$$

где $s_{0}=0$.

Перейдем теперь к нашему фактор-комплексу $\bar{C}_{k}^{q}$. Аналогично случаю диагонального комплекса мы можем определить фильтрацию в пространстве $\bar{C}_{k}^{q}$ подпространствами $\left(\bar{C}_{k}^{q}\right)_{p}=\mathrm{j}_{k}\left(\widetilde{C}_{k, p}^{q} /\left(\widetilde{C}_{k, p}^{q} \cap\right.\right.$ $\left.\left.\cap \widetilde{C}_{k-1}^{q}\right)\right)$, где $\mathrm{j}_{k}$ - проекция на фактор-комплекс. Обозначим соответствующую спектральную последовательность через $\left(\bar{E}_{k, r}^{m, q-m}, \bar{\delta}_{k, r}\right)$.

Рассмотрим спектральные последовательности $\left(\bar{E}_{k, r}^{m, q-m}, \bar{\delta}_{k, r}\right)$ и $\left(\widetilde{E}_{k, r}^{m, q-m}, \widetilde{\delta}_{k, r}\right)$. Обозначим через $B_{k}$ подмножество $\left(S^{1}\right)^{k}$, состоящее из таких точек $\left(t_{1}, \ldots, t_{k}\right)$, что среди точек $t_{1}, \ldots, t_{k}$ есть, по крайней мере, две одинаковые точки или две точки отличающиеся знаком. Пусть $A_{k}=B_{k} \bigcup\{0, \pi\} \times$ $\times T^{k-1} \bigcup S^{1} \times\{0, \pi\} \times T^{k-2} \bigcup T^{2} \times\{0, \pi\} \times T^{k-3} \bigcup \ldots \bigcup T^{k-1} \times\{0, \pi\}$. Справедлива следующая лемма.

Лемма 6. Первый член спектральной последовательности $\left(\bar{E}_{k, r}^{m, q-m}, \bar{\delta}_{k, r}\right)$ определяется равенствами

$$
\begin{gathered}
\bar{E}_{k, 1}^{m, q-m}=0, \quad m \neq 3 k, \quad q \neq 2 k, 2 k+1, \ldots, 3 k, \\
\bar{E}_{k, 1}^{3 k, q-3 k} \cong\left\{\sum \varphi\left(t_{1}, \ldots, t_{k}\right)\left(\widetilde{\lambda}_{1} \otimes \ldots \otimes \widetilde{\lambda}_{k}\right)\right\}, \quad q=2 k, 2 k+1, \ldots, 3 k,
\end{gathered}
$$

где многочлены $\lambda_{i} \in P_{m_{i}}^{s_{i}}$ равнь либо $\lambda^{2}$, либо $\lambda^{3}, \varphi-$ обобщенная функция на $T^{k}$, заданная с точностью до обобщенных функций, сосредоточенных на множестве $A_{k}$, четная (нечетная) по $i$-му аргументу, если $m_{i}-s_{i}-$ четно (нечетно).

Доказательство. Аналогично случаю диагонального подкомплекса всякий элемент пространства $\widetilde{E}_{k, 0}^{m, q-m}$ можно представить в виде коцепи $\sum \varphi\left(\widetilde{\lambda}_{1} \otimes \ldots \otimes \widetilde{\lambda}_{k}\right)$, значение которой на векторных полях определяется так же как в формуле (13) с заменой $\lambda_{i}$ на $\widetilde{\lambda}_{i}$ и $\varphi-$ обобщенная функция на 
$k$-мерном торе $T^{k}$, четность которой по $i$-му аргументу зависит от степени многочлена $\lambda_{i}$ так же как и в формулировке леммы. Обобщенная функция $\varphi$ задана с точностью до обобщенной функции, сосредоточенной на множестве $B_{k}$.

Элементы пространства $\bar{E}_{k, 0}^{m, q-m}$ представляются в виде $\sum \varphi\left(\widetilde{\lambda}_{1} \otimes \ldots \otimes \widetilde{\lambda}_{k}\right)$, где $\varphi-$ обобщенная функция на $\left(S^{1}\right)^{k}$, заданная с точностью до обобщенных функций, сосредоточенных на замкнутом подмножестве $A_{k}$, четность которых по $i$-му аргументу зависит от степени многочлена $\lambda_{i}$.

Из формулы (15) по аналогии со случаем диагонального комплекса следует, что дифференциал $\bar{\delta}_{k, 0}$ действует по формуле

$$
\bar{\delta}_{k, 0}\left(\varphi\left(\widetilde{\lambda}_{1} \otimes \ldots \otimes \widetilde{\lambda}_{k}\right)\right)=\sum_{i=1}^{k}(-1)^{s_{1}+\ldots+s_{i-1}} \varphi\left(\widetilde{\lambda}_{1} \otimes \ldots \otimes \widetilde{\nabla \lambda}_{i} \otimes \ldots \otimes \widetilde{\lambda}_{k}\right) .
$$

Таким образом, действие дифференциала $\bar{\delta}_{k, 0}$ сводится к действию дифференциала $\nabla$ на пространствах многочленов $P_{m_{i}}^{s_{i}}$, откуда следует утверждение леммы.

Элементы пространств $\bar{E}_{k, 1}^{3 k, q-3 k}$ удовлетворяют условиям инвариантности (14). Дифференциал $\bar{\delta}_{k, 1}$ определяется из формул (10) и (16).

Обозначим через $\left(D^{*}\left(T^{k}\right), b\right)$ комплекс потоков де Рама на $k$-мерном торе $T^{k}$. Поток степени $q$ можно представить в виде $\sum_{1 \leq i_{1}<\ldots<i_{q} \leq k} \varphi_{i_{1}, \ldots i_{q}} d t_{i_{1}} \wedge \ldots \wedge d t_{i_{q}}$. Значения потоков на дифференциальных формах определяются следующим образом:

$$
\begin{gathered}
\sum_{1 \leq i_{1}<\ldots<i_{q} \leq k} \varphi_{i_{1}, \ldots i_{q}} d t_{i_{1}} \wedge \ldots \wedge d t_{i_{q}}\left(\sum_{1 \leq i_{1}<\ldots<i_{k-q} \leq k} f_{i_{1}, \ldots i_{k-q}} d t_{i_{1}} \wedge \ldots \wedge d t_{i_{k-q}}\right)= \\
=\sum_{1 \leq i_{1}<\ldots<i_{q} \leq k} \varphi_{i_{1}, \ldots i_{q}}\left(f_{j_{1}, \ldots j_{k-q}}\right),
\end{gathered}
$$

где $j_{1} \neq i_{1}, \ldots, i_{q}, \ldots, j_{k-q} \neq i_{1}, \ldots, i_{q}$ и $j_{1}<j_{2}<\ldots<j_{k-q}$.

Обозначим, через $\left(D^{*}\left(X^{q}\right), b\right)$ комплекс потоков де Рама на орбифолде $X^{k}$ или, что то же самое, комплекс потоков, инвариантных относительно действия группы $\left(\mathbb{Z}_{2}\right)^{k}$ на $k$-мерном торе. Потоки из $D^{*}\left(X^{k}\right)$ можно представить в таком же виде, что и потоки на торе с дополнительными условиями на обобщенные функции: $\varphi_{i_{1}, \ldots i_{q}}\left(t_{1}, \ldots, t_{k}\right)$ - четная по аргументам $t_{i_{1}}, \ldots, t_{i_{q}}$ и нечетная по остальным аргументам.

Обозначим через $\left(D_{A_{k}}^{*}\left(T^{k}\right), b\right)$ комплекс потоков на $k$-мерном торе, сосредоточенных на множестве $A_{k}$, а через $\left(D_{A_{k}}^{*}\left(X^{k}\right), b\right)-$ комплекс потоков, сосредоточенных на $A_{k}$ и инвариантных относительно действия группы $\left(\mathbb{Z}_{2}\right)^{k}$.

Аналогично случаю диагонального комплекса мы можем построить отображение $\gamma_{k}: \bar{E}_{k, 1}^{3 k, q-3 k} \rightarrow$ $\rightarrow D^{3 k-q}\left(X^{k}\right) / D_{A_{k}}^{3 k-q}\left(X^{k}\right)$, которое будет изоморфизмом комплекса $\bar{E}_{k, 1}^{3 k, q-3 k}$ и некоторого подкомплекса в $D^{3 k-q}\left(X^{k}\right) / D_{A_{k}}^{3 k-q}\left(X^{k}\right)$, который определяется из условия инвариантности (14). Применяя рассуждения, аналогичные рассуждениям для диагонального комплекса, получим, что

$$
H^{q}\left(D^{*}\left(T^{k}\right) / D_{A_{k}}^{*}\left(T^{k}\right)\right) \cong H^{q}\left(T^{q} \backslash A_{k}\right) .
$$

Пространство $T^{k} \backslash A_{k}$ представляет собой несвязное объединение $2^{k} k$ ! частей, каждая из которых изоморфна $\mathbb{R}$. Поэтому $H^{0}\left(T^{k} \backslash A_{k}\right) \cong \mathbb{R}^{2^{k} k !}$ и $H^{q}\left(T^{k} \backslash A_{k}\right)=0$, при $q \geq 0$. Применяя условие инвариантности относительно действия группы $\mathbb{Z}_{2}^{k}$ к соответствующим коциклам в $H^{0}\left(D^{*}\left(T^{k}\right) / D_{A_{k}}^{*}\left(T^{k}\right)\right)$, получим $k$ ! различных классов когомологий в $\left(H^{0}\left(D^{*}\left(T^{k}\right) / D_{A_{k}}^{*}\left(T^{k}\right)\right)_{I n v}\right.$. Применяя к ним условие инвариантности (14), получим одну образующуюв в $\bar{E}_{k, 2}^{3 k,-3 k}$, представленную классом коцикла $\underbrace{\beta \wedge \ldots \wedge \beta}_{k}$, где коцикл $\beta$ определяется равенством (3). Коцикл $\underbrace{\beta \wedge \ldots \wedge \beta}_{k}$ является коциклом всего комплекса $C^{*}(\mathscr{U}(X))$, поэтому дифференциалы $\bar{\delta}_{k, q}=0$ при $q \geq 2$ и $\bar{E}_{k, 2}=\bar{E}_{k, 3}=\ldots=\bar{E}_{k, \infty}$. Таким образом, мы доказали следующую теорему.

Теорема 8. $H^{2 k}\left(\bar{C}_{k}^{*}\right) \cong \mathbb{R} u H^{q}\left(\bar{C}_{k}^{*}\right)=0$ при $q \neq 2 k$, где образующая степени $2 k-\kappa л а с с$ коцикла $\underbrace{\beta \wedge \ldots \wedge \beta}_{k}$, коцикл $\beta$ определяется равенством (3). 


\section{2. Когомологии комплекса $\left(\widetilde{C}_{0, \pi}^{q}\right)_{k}$}

Теорема 9. $H^{2 k-1}\left(\left(\widetilde{C}_{0, \pi}^{*}\right)_{k}\right) \cong \mathbb{R} \oplus \mathbb{R}, H^{2 k-2}\left(\left(\widetilde{C}_{0, \pi}^{*}\right)_{k}\right) \cong \mathbb{R} u H^{q}\left(\left(\widetilde{C}_{0, \pi}^{*}\right)_{k}\right)=0, n p u q \neq 2 k-1,2 k-2$, где образующие степени $k-1-\kappa л а с с ы к о ц и к л о в ~ \alpha_{1} \wedge \underbrace{\beta \wedge \ldots \wedge \beta}_{k-1} u \alpha_{2} \wedge \underbrace{\beta \wedge \ldots \wedge \beta}_{k-1}$, образующая степени $k-2-$ класс коциикла $\alpha_{1} \wedge \alpha_{2} \underbrace{\beta \wedge \ldots \wedge \beta}_{k-2}$. Коциилль $\alpha_{1}, \alpha_{2}$ и $\beta$ определяются равенствами (2) и (3).

Доказательство. Будем доказывать теорему индукцией по $k$. Пусть сначала $k=2$. Обозначим через $\widetilde{C}_{\wedge}^{q}$ комплекс $\bigoplus_{p+r=q}\left(\widetilde{C}_{0}^{p} \otimes \widetilde{C}_{0}^{r} \oplus \widetilde{C}_{\pi}^{p} \otimes \widetilde{C}_{\pi}^{r}\right)$. Тогда $\left(\widetilde{C}_{0, \pi}^{q}\right)_{2} \cong\left(\bigoplus_{p+r=q}\left(\left(\widetilde{C}_{0, \pi}^{p}\right) \Delta \otimes \widetilde{C}_{\triangle}^{r}\right)\right) / \widetilde{C}_{\wedge}^{q}$. Когомологии комплекса $\widetilde{C}_{\wedge}^{q}$ тривиальны, поэтому $H^{q}\left(\left(\widetilde{C}_{0, \pi}^{*}\right)_{2}\right) \cong H^{q}\left(\underset{p+r=*}{\bigoplus_{0, \pi}}\left(\left(\widetilde{C}_{0, \pi}^{p}\right) \Delta \otimes \widetilde{C}_{\triangle}^{r}\right)\right)$. Получаем утверждение теоремы для случая $k=2$. Теперь, используя найденные когомологии подкомплекса $\left(\widetilde{C}_{0, \pi}^{*}\right)_{2}$, когомологии фактор-комплекса $\bar{C}_{2}^{*}$, найдем когомологии комплекса $\widetilde{C}_{2}^{*} / \widetilde{C}_{1}^{*}$ с помощью длинной точной последовательности когомологий (12) при $k=2$ :

$$
\left.\ldots \rightarrow H^{i}\left(\left(\widetilde{C}_{0, \pi}^{*}\right)_{2}\right) \rightarrow H^{i}\left(\widetilde{C}_{2}^{*} / \widetilde{C}_{1}^{*}\right) \rightarrow H^{i}\left(\bar{C}_{2}^{*}\right) \rightarrow H^{i+1}\left(\widetilde{C}_{0, \pi}^{*}\right)_{2}\right) \rightarrow H^{i+1}\left(\widetilde{C}_{2}^{*} / \widetilde{C}_{1}^{*}\right) \rightarrow \ldots
$$

Получим:

$$
\begin{gathered}
H^{2}\left(\widetilde{C}_{2}^{*} / \widetilde{C}_{1}^{*}\right) \cong \mathbb{R}, \quad H^{3}\left(\widetilde{C}_{2}^{*} / \widetilde{C}_{1}^{*}\right) \cong \mathbb{R} \oplus \mathbb{R}, \quad H^{4}\left(\widetilde{C}_{2}^{*} / \widetilde{C}_{1}^{*}\right) \cong \mathbb{R}, \\
H^{q}\left(\widetilde{C}_{2}^{*} / \widetilde{C}_{1}^{*}\right)=0, \quad q \neq 2,3,4 .
\end{gathered}
$$

Двумерная образующая - класс коцикла $\alpha_{1} \wedge \alpha_{2}$, трехмерная образующая - классы коциклов $\alpha_{1} \wedge \beta$, $\alpha_{2} \wedge \beta$, четырехмерная образующая - класс коцикла $\beta \wedge \beta$.

Пусть $k=3$. Тогда

$$
\left(\widetilde{C}_{0, \pi}^{q}\right)_{3} \cong\left(\bigoplus_{p+r=q}\left(\left(\widetilde{C}_{0, \pi}^{p}\right) \Delta \otimes \widetilde{C}_{2}^{r}\right)\right) /\left(\bigoplus_{p+r=q}\left(\left(\widetilde{C}_{0, \pi}^{p}\right) \Delta \otimes \widetilde{C}_{\triangle}^{r}\right)\right) .
$$

Найдем когомологии комплекса $\widetilde{C}_{2}^{*}$ из длинной точной последовательности когомологий:

$$
\ldots \rightarrow H^{i}\left(\widetilde{C}_{1}^{*}\right) \rightarrow H^{i}\left(\widetilde{C}_{2}^{*}\right) \rightarrow H^{i}\left(\widetilde{C}_{2}^{*} / \widetilde{C}_{1}^{*}\right) \rightarrow H^{i+1}\left(\widetilde{C}_{1}^{*}\right) \rightarrow H^{i+1}\left(\widetilde{C}_{2}^{*}\right) \rightarrow \ldots
$$

Пространство когомологий комплекса $\bigoplus_{p+r=q}\left(\left(\widetilde{C}_{0, \pi}^{p}\right) \triangle \otimes \widetilde{C}_{2}^{r}\right)-$ тензорное произведение пространств когомологий комплексов $\left(\widetilde{C}_{0, \pi}^{q}\right) \triangle$ и $\widetilde{C}_{2}^{q}$. Найдем когомологии комплекса $\left(\widetilde{C}_{0, \pi}^{q}\right)_{3}$ из длинной точной последовательности комологий комплекса $\bigoplus_{p+r=q}\left(\left(\widetilde{C}_{0, \pi}^{p}\right) \Delta \otimes \widetilde{C}_{2}^{r}\right)$, подкомплекса $\bigoplus_{p+r=q}\left(\left(\widetilde{C}_{0, \pi}^{p}\right) \triangle \otimes \widetilde{C}_{\triangle}^{r}\right)$ и фактор-комплекса $\left(\widetilde{C}_{0, \pi}^{q}\right)_{3}$ и получим утверждение для случая $k=3$.

Теперь, используя длинную точную последовательность (12) при $k=3$, мы можем вычислить когомологии комплекса $\widetilde{C}_{3}^{*} / \widetilde{C}_{2}^{*}$.

Пусть $k=l$, тогда

$$
\left(\widetilde{C}_{0, \pi}^{q}\right)_{l} \cong\left(\bigoplus_{p+r=q}\left(\left(\widetilde{C}_{0, \pi}^{p}\right)_{\Delta} \otimes \widetilde{C}_{l-1}^{r}\right)\right) /\left(\bigoplus_{p+r=q}\left(\left(\widetilde{C}_{0, \pi}^{p}\right)_{\Delta} \otimes \widetilde{C}_{l-2}^{r}\right)\right) .
$$

Считая утверждение теоремы верным для случая $k \leq l-1$, найдем сначала когомологии комплекса $\widetilde{C}_{l-1}^{*} / \widetilde{C}_{l-2}^{*}$, используя длинную точную последовательность когомологий (12) при $k=l-1$. Далее, из длинной точной последовательности когомологий комплекса $\widetilde{C}_{l-1}^{*}$, подкомплекса $\widetilde{C}_{l-2}^{*}$ и фактор-комплекса $\widetilde{C}_{l-1}^{*} / \widetilde{C}_{l-2}^{*}$ найдем когомологии комплекса $\widetilde{C}_{l-1}^{*}$. Пространство когомологий комплекса $\underset{p+r=q}{\bigoplus}\left(\left(\widetilde{C}_{0, \pi}^{p}\right) \Delta \otimes \widetilde{C}_{l-1}^{r}\right)-$ тензорное произведение пространств когомологий комплексов $\left(\widetilde{C}_{0, \pi}^{q}\right)_{\Delta}$ и $\widetilde{C}_{l-1}^{q}$, пространство когомологий комплекса $\bigoplus_{p+r=q}\left(\left(\widetilde{C}_{0, \pi}^{p}\right) \triangle \otimes \widetilde{C}_{l-2}^{r}\right)-$ тензорное произведение пространств когомологий комплексов $\left(\widetilde{C}_{0, \pi}^{q}\right)_{\Delta}$ и $\widetilde{C}_{l-2}^{q}$. Найдем когомологии комплекса $\left(\widetilde{C}_{0, \pi}^{q}\right)_{l}$ из 
длинной точной последовательности когомологий комплекса $\underset{p+r=q}{\bigoplus}\left(\left(\widetilde{C}_{0, \pi}^{p}\right) \triangle \otimes \widetilde{C}_{l-1}^{r}\right)$, подкомплекса $\bigoplus_{p+r=q}\left(\left(\widetilde{C}_{0, \pi}^{p}\right) \triangle \otimes \widetilde{C}_{l-2}^{r}\right)$ и фактор-комплекса $\left(\widetilde{C}_{0, \pi}^{q}\right)_{l}$, что завершает доказательство теоремы.

В ходе доказательства теоремы 9 нам приходилось вычислять когомологии комплексов $\widetilde{C}_{k}^{*} / \widetilde{C}_{k-1}^{*}$, поэтому попутно мы доказали теорему 7.

Доказательство теоремы 2. Когомологии комплекса $\widetilde{C}_{k}^{q} / \widetilde{C}_{k-1}^{q}-$ первый член спектральной последовательности $\left(\widetilde{E}_{r}^{k, q-k}, \delta_{r}\right)$ комплекса $\mathrm{i}\left(C^{q}(\mathscr{U}(X))\right)$, при этом $\delta_{r}=0$ при $r \geq 1$. Тогда

$$
\widetilde{E}_{1}^{k, q-k}=\widetilde{E}_{2}^{k, q-k}=\ldots=\widetilde{E}_{\infty}^{k, q-k}
$$

Рассмотрим подкомплекс комплекса $\mathrm{i}\left(C^{q}(\mathscr{U}(X))\right)$, мультипликативно порожденный коциклами $\alpha_{1}$, $\alpha_{2}$ и $\beta$. Обозначим его через $C_{\alpha, \beta}^{q}$. Ограничим фильтрацию комплекса $\mathrm{i}\left(C^{q}(\mathscr{U}(X))\right)$ подкомплексами $\widetilde{C}_{k}^{q}$ на подкомплекс $C_{\alpha, \beta}^{q}$ и рассмотрим соответствующую спектральную последовательность $\left(\widetilde{E}_{r, \alpha, \beta}^{k, q-k}, \delta_{r, \alpha, \beta}\right)$. Получим, что $\widetilde{E}_{\infty}^{k, q-k} \cong \widetilde{E}_{\infty, \alpha, \beta}^{k, q-k}$. Тогда $H^{q}\left(\mathrm{i}\left(C^{*}(\mathscr{U}(X))\right)\right) \cong H^{q}\left(C_{\alpha, \beta}^{*}\right)$. Алгебра когомологий комплекса $C_{\alpha, \beta}^{q}$ представляет собой тензорное произведение внешней алгебры с двумя образующими степени 1 (классы коциклов $\alpha_{1}$ и $\alpha_{2}$ ) и кольца многочленов с одной образующей степени 2 (класс коцикла $\beta$ ).

В заключение автор выражает благодарность своему научному руководителю, профессору М. В. Лосику за неоценимую помощь в работе.

\section{Библиографический список}

1. Волокитина Е. Ю. О когомологиях алгебры Ли векторных полей на $S_{1} / Z_{2} / /$ Изв. Сарат. ун-та. Нов. сер. Сер. Математика. Механика. Информатика. 2012. Т. 12, вып. 1. С. 8-15.

2. Гельфанд И. М., Фукс Д. Б. Когомологии алгебры Ли касательных векторных полей гладкого многообразия // Функц. анализ. 1969. Т. 3, вып. 3. С. 32-52. 3. Schwartz L. Théorie des distributiones. Paris Hermann, 1951. 418 p.

4. Гельфанд И. М., Виленкин Н. Я. Некоторые приме-

нения гармонического анализа. Оснащенные гильбертовы пространства. М. : Физматгиз, 1961. 472 с.

5. Гельфанд И. М., Шилов Г. Е. Пространства основных и обобщенных функций. М. : Физматгиз, 1958. 308 с.

6. Рам Ж. де. Дифференцируемые многообразия. М. : Изд-во иностр. лит., 1956. 250 с.

7. Годеман Р. Алгебраическая топология и теория пучков. М. : Изд-во иностр. лит., 1961. 320 с.

8. Фоменко А. Т., Фукс Д. Б. Курс гомотопической топологии. М. : Наука, 1989. 528 с.

\section{Cohomology of the Lie Algebra of Vector Fields on Some One-dimensional Orbifold}

\section{E. Y. Volokitina}

Saratov State University, Russia, 410012, Saratov, Astrahanskaya st., 83, evgenia.yu@gmail.com

I. M. Gelfand and D. B. Fuchs have proved that the cohomology algebra of the Lie algebra of vector fields on the unit circle is isomorphic to the tensor product of the polynomial ring with one generator of degree two and the exterior algebra with one generator of degree three. In the present paper the cohomology of the Lie algebra of vector fields on the one-dimensional orbifold $S^{1} / \mathbb{Z}_{2}$ are studied. $S^{1} / \mathbb{Z}_{2}$ is the orbit space under the $\mathbb{Z}_{2}$ group action on the unit circle by reflection in the Ox axis. It has been proved that the cohomology algebra of the Lie algebra of vector fields on the orbifold is isomorphic to the tensor product of the exterior algebra with two generators of degree one and the polynomial ring with one generator of degree two. To prove this result author used the Gelfand-Fuchs method with some modifications.

Key words: orbifold, Lie algebra, cohomology.

\section{References}

1. Volokitina E. Y. Cohomology of Lie algebra of vector fields on $S_{1} / Z_{2}$. Izv. Sarat. Univ. N.S. Ser. Math. Mech. Inform., 2012. vol. 12, iss. 1, pp. 8-15 (in Russian).

2. Gelfand I. M., Fuks D. B. Cohomologies of Lie algebra of tangential vector fields of a smooth manifold. Functional Analysis and Its Applications, 1969, vol. 3, iss. 3 , pp. 194-210.

3. Schwartz L. Théorie des distributiones. Paris, Hermann, 1951, $418 \mathrm{p}$.

4. Gelfand I. M., Vilenkin N. Ja. Generalized Functions, vol. 4: Some Applications of Harmonic Analysis. Rigged Hilbert Spaces. New York, Academic Press, 1964, 384 p. (Rus. ed.: Gelfand I. M., Vilenkin N. Ja. Nekotorye primeneniia garmonicheskogo analiza. Osnashchennye 
gil'bertovy prostranstva. Moscow, Fizmatgiz, 1961, 7. Godeman R. Algebraicheskaia topologiia $i$ teoriia 472 p.) puchkov [Algebraic topology and theory of sheaves].

5. Gelfand I. M., Shilov G. E. Prostranstva osnovnykh $i$ obobshchennykh funktsii [Spaces of test and generalized functions]. Moscow, Fizmatgiz, 1958, 308 p. (in Russian). 6. Rham G. de. Differentsiruemye mnogoobraziia [Differentiable manifolds]. Moscow, Izdatelstvo inostrannoj literatury, 1956, 250 p. (in Russian). Moscow, Izdatelstvo inostrannoj literatury, 1961, 320 p. (in Russian).

8. Fomenko A. T., Fuks D. B. Kurs gomotopicheskoi topologii [A course in homotopy topology]. Moscow, Nauka, 1989, 528 p. (in Russian).

УДК 501.1

\title{
СИСТЕМА ДИРАКА С НЕДИФФЕРЕНЦИРУЕМЫМ ПОТЕНЦИАЛОМ И АНТИПЕРИОДИЧЕСКИМИ КРАЕВЫМИ УСЛОВИЯМИ
}

\author{
В. В. Корнев ${ }^{1}$, А. П. Хромов ${ }^{2}$
}

\begin{abstract}
${ }^{1}$ Кандидат фризико-математических наук, доцент кафредры диффференциальных уравнений и прикладной математики, Саратовский государственный университет им. Н. Г. Чернышевского, KornevVV@info.sgu.ru

2 Доктор фризико-математических наук, заведующий кафредрой диффреренциальных уравнений и прикладной математики, Саратовский государственный университет им. Н. Г. Чернышевского, KhromovAP@info.sgu.ru

В работе рассматривается система Дирака с антипериодическими краевыми условиями и с комлекснозначным непрерывным потенциалом. Предложен новый метод исследования спектральных свойств этой краевой задачи. Метод базируется на фрормулах типа операторов преобразования и является элементарным и простым. С его помощью получена уточненная асимптотика собственных значений и доказано, что система собственных и присоединенных функций образует базис Рисса со скобками в пространстве квадратично суммируемых двумерных вектор-фрункций, так как собственные значения могут быть кратными. Исследуется также структура проекторов Рисса. Полученные результаты можно использовать в смешанной задаче для уравнения с частными производными первого порядка с инволюцией.
\end{abstract}

Ключевые слова: система Дирака, спектр, асимптотика, базис Рисса.

Рассмотрим на отрезке $[0,1]$ систему Дирака:

$$
\begin{gathered}
y_{1}^{\prime}(x)-q_{2}(x) y_{2}(x)=\lambda y_{1}(x), \\
y_{2}^{\prime}(x)-q_{1}(x) y_{1}(x)=-\lambda y_{2}(x)
\end{gathered}
$$

с краевыми условиями

$$
y_{1}(0)=-y_{1}(1), \quad y_{2}(0)=-y_{2}(1)
$$

где $q_{j}(x)$ - непрерывные комплекснозначные функции.

В работе [1] предложен новый метод исследования спектральных свойств системы (1), (2) в случае периодических краевых условий. В данной работе на основе этого метода подобное исследование осуществляется в случае антипериодических краевых условий (3). Метод базируется на формулах типа операторов преобразования (см. также [2, с. 30]), является элементарным и весьма простым. В качестве приложения дается новое доказательство теоремы П. Джакова, Б. С. Митягина $[3,4]$ о базисах Рисса. Как и в периодическом случае, в антипериодическом случае возможна кратность собственных значений. Полученные результаты могут быть также использованы в смешанной задаче для уравнения с частными производными первого порядка с инволюцией [5].

\section{1. АСИМПТОТИКА СОБСТВЕННЫХ ЗНАЧЕНИЙ И ПОЛНОТА СИСТЕМЫ СОБСТВЕННЫХ И ПРИСОЕДИНЕННЫХ ФУНКЦИЙ}

Имеет место следующая асимптотика решений системы (1), (2):

Лемма 1. Система (1), (2) в области $\operatorname{Re} \lambda \geq-h, h>0$, при больших $|\lambda|$ имеет фундаментальную матрицу решений $Y(x, \lambda)=\left(y_{i j}(x)\right)_{1}^{2}$ с асимптотикой

$$
Y(x, \lambda)=(E+o(1)) e^{\lambda D x},
$$

где $E=\operatorname{diag}(1,1), D=\operatorname{diag}(1,-1)$, o(1) $\rightarrow 0$ при $|\lambda| \rightarrow \infty$ равномерно по $x \in[0,1] u \arg \lambda, y_{i j}(x)-$ аналитичны по $\lambda$. 